\title{
Article \\ A Full Description of $\omega$-Limit Sets of Cournot Maps Having Non-Empty Interior and Some Economic Applications
}

\author{
Antonio Linero-Bas ${ }^{1, *,+(\mathbb{D})}$ and María Muñoz-Guillermo ${ }^{2,+} \mathbb{D}$ \\ 1 Departamento de Matemáticas, Campus de Espinardo, Universidad de Murcia, 30100 Murcia, Spain \\ 2 Departamento de Matemática Aplicada y Estadística, Universidad Politécnica de Cartagena, \\ Antiguo Hospital de Marina, 30202 Cartagena, Murcia, Spain; maria.mg@upct.es \\ * Correspondence: lineroba@um.es \\ + These authors contributed equally to this work.
}

check for

updates

Citation: Linero-Bas, A.;

Muñoz-Guillermo, M. A Full

Description of $\omega$-Limit Sets of

Cournot Maps Having Non-Empty Interior and Some Economic Applications. Mathematics 2021, 9, 452. https://doi.org/10.3390/ math9040452

Academic Editor: Mehdi Salimi

Received: 28 December 2020

Accepted: 16 February 2021

Published: 23 February 2021

Publisher's Note: MDPI stays neutral with regard to jurisdictional claims in published maps and institutional affiliations.

Copyright: (c) 2021 by the authors. Licensee MDPI, Basel, Switzerland. This article is an open access article distributed under the terms and conditions of the Creative Commons Attribution (CC BY) license (https:// creativecommons.org/licenses/by/ $4.0 /)$.

\begin{abstract}
Given a continuous Cournot map $F(x, y)=\left(f_{2}(y), f_{1}(x)\right)$ defined from $I^{2}=[0,1] \times[0,1]$ into itself, we give a full description of its $\omega$-limit sets with non-empty interior. Additionally, we present some partial results for the empty interior case. The distribution of the $\omega$-limits with non-empty interior gives information about the dynamics and the possible outputs of each firm in a Cournot model. We present some economic models to illustrate, with examples, the type of $\omega$-limits that appear.
\end{abstract}

Keywords: $\omega$-limit set; Cournot map; non-empty interior; Cournot duopoly model; topological mixing map

MSC: 37E05; 37N40; 91B54

\section{Introduction}

Topological structure and classification of $\omega$-limits for general compact metric spaces remain an important problem and, in many aspects, an unknown subject. In order to better understand the main goal pursued in this paper in relation to the description of certain $\omega$-limit sets for Cournot maps, and their presence in the numeric simulations of several economic models, let us first present the needed notions and their associated notation.

Given a compact metric space $X$, let $C(X, X)$ denote the set of continuous maps from $X$ into itself. The interior of a subset $Y \subset X$ is denoted by $\operatorname{Int}(Y)$. We say that $Y \subset X$ is a nowhere dense set whenever the interior of its closure is empty. Let $\varphi \in C(X, X)$. For $n \in \mathbb{N}$ we put $\varphi^{n}=\varphi \circ \varphi^{n-1}$, with $\varphi^{0}=$ Identity. The orbit of a point $x \in X$ through $\varphi$ is $\operatorname{Orb}_{\varphi}(x)=\left\{\varphi^{n}(x)\right\}_{n=0}^{\infty}$. We say that $Y \subset X$ is n-periodic if $\varphi^{n}(Y)=Y$ and $\varphi^{k}(Y) \neq Y$ for $0<k<n$; in this case, $\operatorname{Orb}_{\varphi}(Y)=Y \cup \varphi(Y) \cup \ldots \cup \varphi^{n-1}(Y)$ is the orbit of $Y$. If $Y=\{x\}$ we say that it is a n-periodic point. The period of $x$ is denoted by $\operatorname{ord}_{\varphi}(x)$. When $X=I:=[0,1]$ is the unit interval and $Y$ is a subinterval of $I$, we have an n-periodic subinterval. If $X=I^{2}$, and $Y=J_{1} \times J_{2}$, with $J_{i}$ subintervals of $I, i=1,2$, we have an n-periodic rectangle.

The set of limit points of $\operatorname{Orb}_{\varphi}(x)$ (i.e., its accumulation points or elements $y$ in $X$, for which there exists an infinite subsequence $n_{1}<n_{2}<\ldots<n_{j}<\ldots$ of positive integers such that $\varphi^{n_{j}}(x)$ converges to $y$ ), is called the $\omega$-limit set of $x$ by $\varphi$, and we denote it by $\omega_{\varphi}(x)$. For any $x \in X, \omega_{\varphi}(x)$ is non-empty, closed and strongly invariant $\left(\varphi\left(\omega_{\varphi}(x)\right)=\omega_{\varphi}(x)\right)$ (see [1]). Moreover, for any $n \in \mathbb{N}$

$$
\omega_{\varphi}(x)=\bigcup_{j=0}^{n-1} \omega_{\varphi^{n}}\left(\varphi^{j}(x)\right) .
$$

We say that $\varphi$ is transitive if, given two non-empty open sets $U, V \subset X$, there is $n_{0} \in \mathbb{N}$ such that $\varphi^{n_{0}}(U) \cap V \neq \varnothing$. If there is $k_{0} \in \mathbb{N}$ such that $\varphi^{n}(U) \cap V \neq \varnothing$ for all $n \geq k_{0}$, then $\varphi$ 
is called a (topologically) mixing map. Observe that topologically mixing implies transitivity (see [2] for a detailed explanation on properties of these types of maps). Moreover, $\varphi$ is transitive if and only if there exists a point $x \in X$ with $\omega_{\varphi}(x)=X$ (see [1]).

The pair $(X, \varphi)$ is called a discrete dynamical system. Given such a system, the main objective is to know the asymptotic behavior of the orbits $\operatorname{Orb}_{\varphi}(x)$ for all $x \in X$ and to analyze the topological structure of their $\omega$-limit sets. This is a difficult problem, and we restrict our attention to a particular class of continuous two-dimensional maps.

Given $f \in C(I, I), I=[0,1]$, the topological structure of an $\omega$-limit set $L$ of $f$ is well known: either $L$ is a nowhere dense set or $L=\bigcup_{i=1}^{n} J_{i}$, where $J_{i}$ are non-degenerate closed subintervals of $I$ such that $f\left(J_{i}\right)=J_{i+1(\bmod n)}$ and $J_{i} \cap J_{k}=\varnothing$ if $i \neq k$ (for a proof, see [1]). Conversely, any set of the above forms can be realized as an $\omega$-limit set for a suitable continuous interval map (see [3] or [4]).

Nevertheless, the description of $\omega$-limit sets for continuous maps $f: I^{n} \rightarrow I^{n}$, with $n \geq 2$, is an open problem. In this direction, only a few results are known (see for instance, [5-9]). It seems to be more reasonable to focus our attention on special classes of two-dimensional continuous maps.

Following [10], we are interested in describing the topological structure of $\omega$-limit sets of a particular class of $C\left(I^{2}, I^{2}\right)$ called antitriangular maps or Cournot maps. We say that $F \in C\left(I^{2}, I^{2}\right)$ is an antitriangular map (or Cournot map) if

$$
F(x, y)=\left(f_{2}(y), f_{1}(x)\right),
$$

with $f_{i} \in C(I, I), i=1$,2. It is immediate to see that for any $n \geq 0$ it holds

$$
\begin{gathered}
F^{2 n}(x, y)=\left(\left(f_{2} \circ f_{1}\right)^{n}(x),\left(f_{1} \circ f_{2}\right)^{n}(y)\right), \\
F^{2 n+1}(x, y)=\left(\left(f_{2} \circ\left(f_{1} \circ f_{2}\right)^{n}\right)(y),\left(f_{1} \circ\left(f_{2} \circ f_{1}\right)^{n}\right)(x)\right) .
\end{gathered}
$$

The set of antitriangular maps on $I^{2}$ will be denoted by $C_{A}\left(I^{2}, I^{2}\right)$.

This type of two-dimensional map appears closely related to an economical process called the Cournot duopoly (see [11]), in which two firms produce an identical good, and in each step they try to obtain the maximum profit according to the decision of the opposite firm in the last step. This economical process has been profusely studied in the literature (for instance, see [12-17],...).

The following result gives a description of $\omega$-limit sets of antitriangular maps.

Theorem 1 ([10] Theorem 2). Let $F \in C_{A}\left(I^{2}, I^{2}\right)$ and let $L=\omega_{F}(x, y),(x, y) \in I^{2}$. Then $L$ can be one of the following types:

1. A finite set (periodic orbit).

2. An infinite nowhere dense set.

3. L is a finite union $\bigcup_{i=1}^{p} R_{i}$, where $R_{i}=I_{i} \times J_{i} \subset I^{2}$ are non-degenerate periodic rectangles of $F$ such that $R_{i} \cup R_{j}$ is not a rectangle and $\operatorname{Int}\left(R_{i}\right) \cap \operatorname{Int}\left(R_{j}\right)=\varnothing$ for $1 \leq i<j \leq p$.

But, as it was highlighted in [10], not every distribution of rectangles in the square can be realized as an $\omega$-limit of a suitable antitriangular map. In this paper, our main goal is to give a full description of $\omega$-limit sets with non-empty interior, and consider some questions about $\omega$-limit sets with empty interior.

This paper is organized as follows. After some preliminary results stated in Section 2, we proceed to develop in Section 3 a full description for the $\omega$-limit sets of Cournot maps $F(x, y)=\left(f_{2}(y), f_{1}(x)\right)$ that have a non-empty interior. It is worth mentioning that this study is strongly related to the nature of the $\omega$-limit sets associated with the onedimensional maps $f_{2} \circ f_{1}$ and $f_{1} \circ f_{2}$, in connection with the property of either being or not being a mixing $\omega$-limit set, see Definition 2. Our analysis will be applied and illustrated with several economic models, such as Puu's duopoly or Matsumoto-Nonaka's model, among others, in Section 4. Next, in Section 5, on the one hand we present some examples 
of $\omega$-limit sets of Cournot maps having empty interior and we show the existence of connected limit sets with empty interior; on the other hand, in the non-empty interior case, we characterize the connected $\omega$-limit sets $\omega_{F}(x, y)$ of Cournot maps $F$ by the inspection of their canonical projections $\pi_{j}\left(\omega_{F}(x, y)\right), j=1,2$, and the intersection of the limit sets $\omega_{F^{2}}(x, y)$ and $\omega_{F^{2}}(F(x, y))$. We also state some open problems with the hope of advancing the comprehension of the empty-interior case of Cournot maps.

\section{Auxiliary Results}

In this section, some auxiliary results are summed up since they will be used later.

Proposition 1 ([10] Proposition 7). Let $F \in C_{A}\left(I^{2}, I^{2}\right)$. The following properties hold for all $Z=\left(z_{1}, z_{2}\right) \in I^{2}$.

1. $\omega_{F}(Z) \subseteq\left[\omega_{f_{2} \circ f_{1}}\left(z_{1}\right) \times \omega_{f_{1} \circ f_{2}}\left(z_{2}\right)\right] \cup\left[\omega_{f_{2} \circ f_{1}}\left(f_{2}\left(z_{2}\right)\right) \times \omega_{f_{1} \circ f_{2}}\left(f_{1}\left(z_{1}\right)\right)\right]$.

2. $\pi_{i}\left(\omega_{F}(Z)\right)=\omega_{f_{j} \circ f_{i}}\left(z_{i}\right) \cup \omega_{f_{j} \circ f_{i}}\left(f_{j}\left(z_{j}\right)\right)$, for $i, j \in\{1,2\}, i \neq j$.

3. $f_{i}\left(\omega_{f_{j} \circ f_{i}}\left(z_{i}\right)\right)=\omega_{f_{i} \circ f_{j}}\left(f_{i}\left(z_{i}\right)\right)$, for $i, j \in\{1,2\}, i \neq j$.

For the next results, recall that by a bitransitive map $f$ we understand a continuous map for which $f$ and $f^{2}$ are transitive.

Lemma 1 ([2] Theorem 6.1.1). Let $f \in C(I, I)$ be transitive. Then either $f$ is bitransitive or there is $c \in(0,1)$ such that $f([0, c])=[c, 1], f([c, 1])=[0, c]$ and both $\left.f^{2}\right|_{[0, c]}$ and $\left.f^{2}\right|_{[c, 1]}$ are bitransitive (clearly the point $c$ is the only fixed point of $f$ ).

Lemma 2 ([2] Theorem 6.1.2). Let $f \in C(I, I)$. The following statements are equivalent:

1. $f$ is bitransitive.

2. $f$ is topologically mixing.

Remark 1. With respect to the last result, we can stress that in the case of a bitransitive map $f: I \rightarrow I$ (therefore, topologically mixing), if $x_{0} \in I$ verifies that $\omega_{f}\left(x_{0}\right)=I$, then $\omega_{f^{s}}\left(x_{0}\right)=I$ for every positive integer s, see ([1], [Prop. 42, Chapter VI]).

The following result allows us to construct $\omega$-limit sets from mixing sets (we will say that an invariant closed set $Y \subset X$ is mixing for a continuous map $\varphi: X \rightarrow X$ whenever $\left.\varphi\right|_{Y}$ is mixing); it can be easily derived from Theorem 6 in [6] where the property is established in the frame of mixing compact $\omega$-limit sets in the Euclidean space $\mathbb{R}^{n}$.

Theorem 2 ([6] Theorem 6). Let $f, g \in C(I, I)$ and let $x \in I$ such that $\omega_{f}(x)$ is mixing. Then for any $y \in I$ there are $x_{1}, y_{1} \in I$ such that $\omega_{f \times g}\left(x_{1}, y_{1}\right)=\omega_{f}(x) \times \omega_{g}(y)$.

The following lemma states the relationship between two $\omega$-limit sets from a continuous map defined in a compact metric space.

Lemma 3 ([10] Lemma 3). Let $\varphi \in C(X, X)$ and suppose that $Y$ and $Z$ are $\omega$-limit sets of $\varphi$ with non-empty interior. Then $\operatorname{Int}(Y) \cap \operatorname{Int}(Z)=\varnothing$ or $Y=Z$.

The case in which $\operatorname{Int}(Y) \cap \operatorname{Int}(Z)=\varnothing$ can be even more specific. The following result deals with interval maps having two different $\omega$-limits sets with non-empty interiors sharing periodic points. According to the structure of the $\omega$-limit sets $L$ with non-empty interior, corresponding to interval maps $f \in C(I, I)$, described in Section 1, we know that $L=\bigcup_{s=1}^{p} I_{s}$ and $f\left(I_{s}\right)=I_{s+1 \bmod (p)}, s=1, \ldots, p$. Then, we say that $L$ is mixing for $f$ whenever the transitive restricted map $\left.f^{p}\right|_{I_{s}}$ is topologically mixing for $s=1, \ldots, p$. Otherwise, $L$ is said to be no mixing. 
Lemma 4. Let $f \in C(I, I)$. Let $A=\bigcup_{i=1}^{p} I_{i}$ and $B=\bigcup_{j=1}^{q} J_{j}$ be two different $\omega$-limit sets of $f$ with non-empty interiors. Suppose $p \geq q$. Suppose $A \cap B \neq \varnothing$ and $\operatorname{Int}(A) \cap B=\varnothing$. Then, either $p=q$ or $p=2 q$, in both cases $A$ is mixing, and the number of connected components of $A \cup B$ is $q$.

Proof. If $A \cap B \neq \varnothing$ and $\operatorname{Int}(A) \cap B=\varnothing$, then it is easily seen that $A$ and $B$ share endpoints, which are evidently periodic points of $f$.

Let $(A \cup B)_{1}<\ldots<(A \cup B)_{c}$ be the $c$ connected components of $A \cup B$ (given $J, K \subset I$, $J<K$ means that $x<y$ for all $x \in J, y \in K$; realize that this number $c$ is finite).

Define

$$
\begin{aligned}
& a_{i}=\operatorname{Card}\left(\left\{1 \leq j \leq p: I_{j} \subset(A \cup B)_{i}\right\}\right), \\
& b_{i}=\operatorname{Card}\left(\left\{1 \leq t \leq q: J_{t} \subset(A \cup B)_{i}\right\}\right) .
\end{aligned}
$$

Obviously, $a_{i_{1}}=a_{i_{2}}, b_{j_{1}}=b_{j_{2}}$ for all $i_{1}, i_{2} \in\{1, \ldots, p\}$ and $j_{1}, j_{2} \in\{1, \ldots, q\}$, since $f$ maps connected components on connected components and $A, B$ are periodic sets of $f$. Moreover, since $p \geq q$, either $a_{1}=b_{1}$ or $a_{1}=b_{1}+1$.

If $b_{1} \geq 2$, it is not restrictive to assume that we have in $(A \cup B)_{1}$ the disposition $I_{i_{1}} \leq J_{j_{1}} \leq I_{i_{2}} \leq J_{j_{2}} \leq \ldots$ for some $i_{\ell} \in\{1, \ldots, p\}, j_{\ell} \in\{1, \ldots, q\}, \ell=1,2, \ldots$ Notice that $c<p$ because $b_{1} \geq 2$ and $q \leq p$. Since $f$ maps connected components on connected components, and $c$ is the number of such connected components, we have $f^{\mathcal{C}}\left((A \cup B)_{1}\right)=(A \cup B)_{1}$. Therefore, being $(A \cup B)_{1}$ a closed interval, there exists a fixed point $x$ of $f^{C}$ in $(A \cup B)_{1}$. Taking into account that $b_{1} \geq 2($ so, $\min \{p, q\} \geq 2)$, we deduce that either $x \in \operatorname{Int}\left(I_{i_{\ell}}\right)$ or $x \in \operatorname{Int}\left(J_{j_{\ell}}\right)$ for some $\ell=1,2, \ldots$ Consequently, either $f^{\mathcal{C}}\left(I_{i_{\ell}}\right)=I_{i_{\ell}}$ or $f^{\mathcal{C}}\left(J_{j_{\ell}}\right)=J_{j_{\ell}}$. The first case is not allowable because $A$ is a $p$-periodic orbit and $c<p$; in the second case, we distinguish two situations: (i) if $J_{j_{\ell}}$ is the endpoint of $(A \cup B)_{1}$ necessarily $f^{\mathcal{C}}\left(I_{i_{\ell}}\right)=I_{i_{\ell}}$, a new contradiction; (ii) if $J_{j_{\ell}}$ is not an endpoint of $(A \cup B)_{1}$, since $b_{1} \geq 2$ we have $J_{j_{\ell}} \leq I_{i_{\ell}} \leq J_{j_{\ell+1}}$, and due to the fact that $f^{c}\left(J_{j_{s}}\right)=J_{j_{s}}$ for all $s$, we deduce that $f^{c}\left(I_{i_{\ell}}\right)=I_{i_{\ell}}$ which again contradicts that $c<p$.

Therefore, we have proved that $b_{1}=1$. This gives $c=q$. Notice that the possibility $a_{1}=b_{1}+1$ can occur.

Due to the structure of the connected components $(A \cup B)_{1}, \ldots,(A \cup B)_{q}$ mentioned above, observe that $f^{p}(x)=x$ for all $x \in A \cap B$ both if $p=q$ (here $\left.(A \cup B)_{s}=I_{i_{s}} \cup J_{j_{s}}\right)$ or $p=2 q$ (where $(A \cup B)_{s}=I_{i_{s}} \cup J_{j_{s}} \cup I_{i_{s+1}}$, with $\left.I_{i_{s}} \leq J_{j_{s}} \leq I_{i_{s+1}}\right)$. This means that each $I_{i}$ has an endpoint as a fixed point of $f^{p}$. Then Lemma 1 and Lemma 2 imply that $A$ is mixing.

Remark 2. Realize that if $p=q$ in the above Lemma 4, we also obtain that $B$ is mixing, but in the case $p=2 q$ it is not guaranteed that $B$ is mixing, because in this situation we only know that its endpoints are fixed by $f^{2 q}$ but we have no information about the action of $f^{q}$ on these endpoints.

To describe the $\omega$-limit sets with non-empty interior, we will use the following results, whose proofs can be consulted in [18]. Among them, we include the characterization of finite limit sets, that is, periodic orbits of Cournot maps; in fact, the movement of periodic rectangles realized as $\omega$-limit sets of Cournot maps can be 'interpreted' as the evolution of periodic points, and in this sense the distribution of periodic points can show us in some cases the possible way to distribute periodic rectangles.

Lemma 5 ([18] Proposition 3.7). Let $F \in C_{A}\left(I^{2}, I^{2}\right)$. Let $M \subset I^{2}$ be a periodic orbit of $F$ and fix $(x, y) \in M$. Then the following statements are equivalent:

1. $M \subseteq \operatorname{Orb}_{f_{2} \circ f_{1}}(x) \times \operatorname{Orb}_{f_{1} \circ f_{2}}(y)$.

2. $M \cap\left(\operatorname{Orb}_{f_{2} \circ f_{1}}(x) \times \operatorname{Orb}_{f_{1} \circ f_{2}}\left(f_{1}(x)\right)\right) \neq \varnothing$.

3. $f_{1}(x) \in \operatorname{Orb}_{f_{1} \circ f_{2}}(y)$.

4. $f_{2}(y) \in \operatorname{Orb}_{f_{2} \circ f_{1}}(x)$. 
Lemma 6 ([18] Proposition 3.8). Let $F \in C_{A}\left(I^{2}, I^{2}\right)$. Let $M \subset I^{2}$ be a periodic orbit of $F$ with $f_{2}(y) \notin \operatorname{Orb}_{f_{2} \circ f_{1}}(x)$ for all $(x, y) \in M$. Then there are $d, k_{1}, k_{2} \in \mathbb{N}$ and there are two finite sets $H, V \subset$ I holding the following properties:

1. $\operatorname{Card}(M)=2 d k_{1} k_{2}, \operatorname{gcd}\left(k_{1}, k_{2}\right)=1, \operatorname{lcm}\left(d k_{1}, d k_{2}\right)=\operatorname{Card}(M) / 2$.

2. For all $(x, y) \in M$, either $d k_{1}=\operatorname{ord}_{f_{2} \circ f_{1}}(x)$ and $d k_{2}=\operatorname{ord}_{f_{1} \circ f_{2}}(y)$, or $d k_{2}=\operatorname{ord}_{f_{2} \circ f_{1}}(x)$ and $d k_{1}=\operatorname{ord}_{f_{1} \circ f_{2}}(y)$.

3. $\operatorname{Card}(H)=k_{1}, \operatorname{Card}(V)=k_{2}$.

4. $\operatorname{Orb}_{F}(H \times V)=M$.

5. For $i=1,2, \pi_{i}\left(F^{j}(H \times V)\right) \cap \pi_{i}\left(F^{\ell}(H \times V)\right)=\varnothing$ for $0 \leq j<\ell<2 d$.

Theorem 3 ([18] Theorem 3.10). Let $M=\left\{\left(x_{i}, y_{i}\right)\right\}_{i=1}^{n}$ be a periodic orbit for $F \in C_{A}\left(I^{2}, I^{2}\right)$. Then

1. If $n \in\{2\} \cup\{2 m+1: m \in \mathbb{N} \cup\{0\}\}, x_{i} \neq x_{j}$ and $y_{i} \neq y_{j}$ for $i \neq j, i, j \in\{1, \ldots, n\}$. See Figure 1.
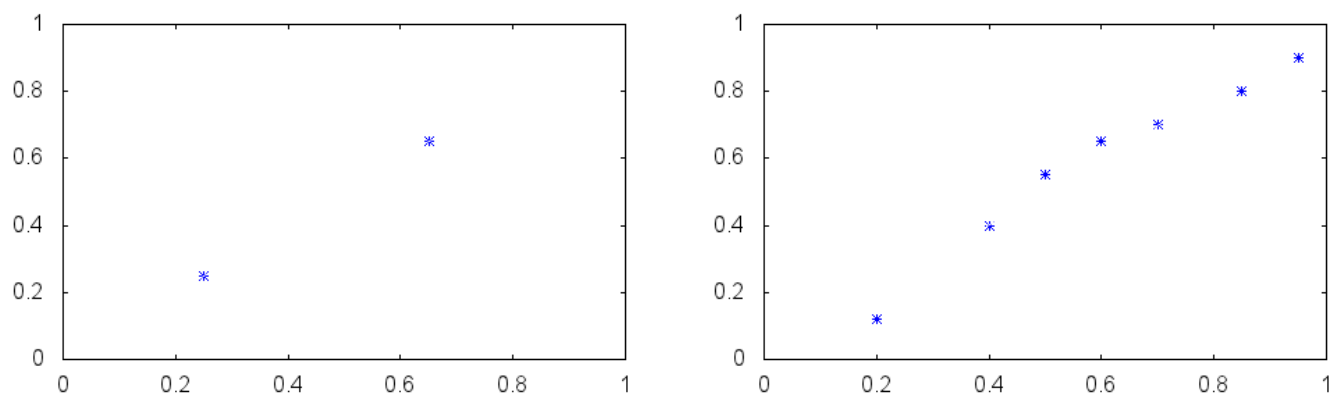

Figure 1. Case (1) in Theorem 3: Two points or an odd number of points.

2. If $n \in\{2 m: m \in \mathbb{N}, m \geq 2\}$, and $M$ satisfies the equivalent conditions of Lemma 5, for any $i \in\{1, \ldots, n\}$ there are unique $j, k \in\{1, \ldots, n\} \backslash\{i\}$ such that $x_{i}=x_{j}$ and $y_{i}=y_{k}$. See Figure 2.

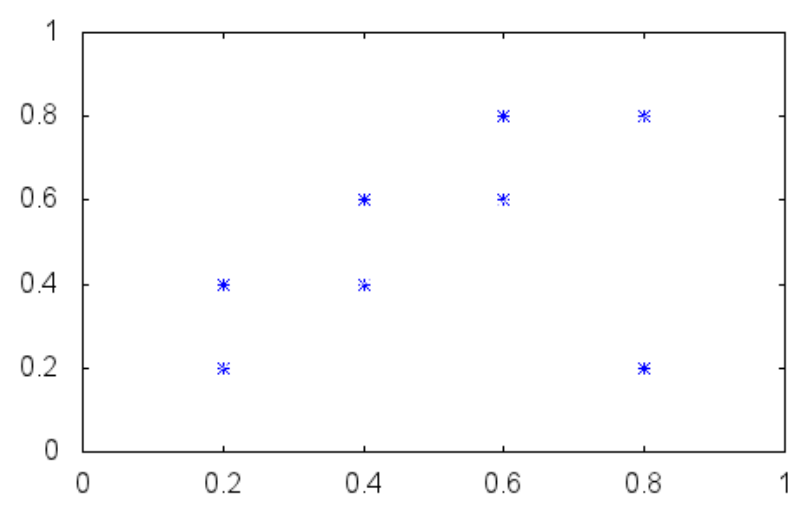

Figure 2. An even number $n$ of points, $n \geq 4$, following the distribution stated in Case (2) of Theorem 3.

3. If $n \in\{2 m: m \in \mathbb{N}, m \geq 2\}$, and $M$ does not satisfy the equivalent conditions of Lemma 5, the distribution of points of $M$ is described by Lemma 6. See Figure 3. 


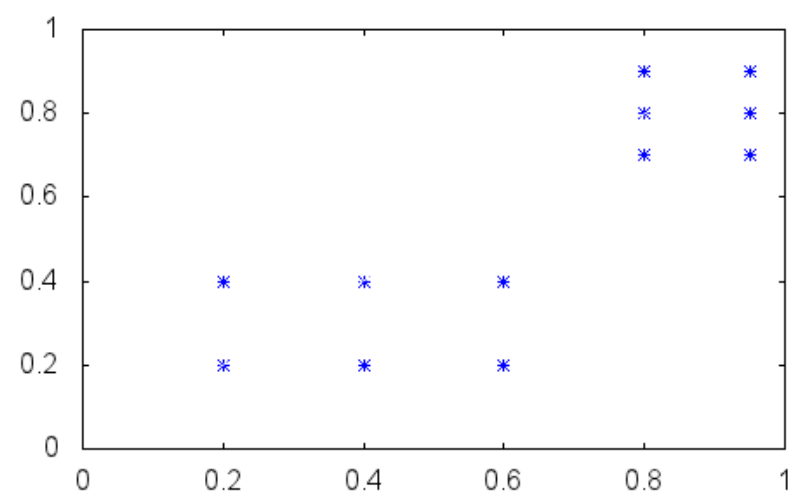

Figure 3. An even number $n$ of points, $n \geq 4$, following the distribution stated in Case (3) of Theorem 3.

\section{Description of the Non-Empty Interior Case}

In this section, the starting point for the analysis lies in fixing the type of $\omega$-limit sets obtained in the one-dimensional case $\left(f_{1} \circ f_{2}\right.$ and $\left.f_{2} \circ f_{1}\right)$ and, then, to study the type of $\omega$-limit sets generated in the two-dimensional case for the antitriangular map $F(x, y)=\left(f_{2}(y), f_{1}(x)\right)$.

Let $F \in C_{A}\left(I^{2}, I^{2}\right)$ be an antitriangular map such that $F(x, y)=\left(f_{2}(y), f_{1}(x)\right)$ where $f_{1}, f_{2} \in C(I, I)$. Let $L=\omega_{F}(x, y)$ be an $\omega$-limit with non empty interior. Then, according to Theorem 1, $L=\cup_{i=1}^{k} R_{i}$, where $k$ is the number of different rectangles in the decomposition. As general observations, we can assume that

$$
\begin{array}{ll}
\omega_{f_{2} \circ f_{1}}(x)=\bigcup_{i=1}^{p} I_{i}=A, & \omega_{f_{1} \circ f_{2}}\left(f_{1}(x)\right)=\bigcup_{i=1}^{p} f_{1}\left(I_{i}\right)=f_{1}(A), \\
\omega_{f_{1} \circ f_{2}}(y)=\bigcup_{j=1}^{q} J_{j}=B, & \omega_{f_{2} \circ f_{1}}\left(f_{2}(y)\right)=\bigcup_{j=1}^{q} f_{2}\left(J_{j}\right)=f_{2}(B),
\end{array}
$$

where $I_{i}$ and $J_{j}$ are closed non-degenerate periodic subintervals of $f_{2} \circ f_{1}$ and $f_{1} \circ f_{2}$ respectively, with periods $p$ and $q$, respectively. In our next development, we put $I_{s}^{\prime}=f_{2}\left(J_{s}\right)$, $J_{t}^{\prime}=f_{1}\left(I_{t}\right), s=1, \ldots, q, t=1, \ldots, p$. Moreover, by Proposition 1 ,

$$
L \subset(A \times B) \cup\left(f_{2}(B) \times f_{1}(A)\right) .
$$

Let $\pi_{1}$ and $\pi_{2}$ be the projection maps on the first and second coordinate, respectively. Let $J$ be an interval appearing in $A \cup B$; for $j=1,2$, we define $p_{j}(J)=\left|\left\{R \subseteq L: \pi_{j}(R)=J\right\}\right|$ the number of rectangles $R$ in $L$, such that $\pi_{j}(R)=J$.

In order to simplify the classification of $\omega$-limit sets with non-empty interior, we consider the following definition.

Definition 1. We say that two $\omega$-limit sets $L_{1}=\cup_{i=1}^{k_{1}} R_{i}$ and $L_{2}=\cup_{j=1}^{k_{2}} \widetilde{R}_{j}$ are equivalent if

1. $k_{1}=k_{2}$;

2. there exists a permutation $\sigma$ of $k=k_{1}=k_{2}$ elements such that $\pi_{j}\left(L_{1}\right)=\pi_{j}\left(L_{2}\right)$ and $p_{j}\left(\pi_{j}\left(\widetilde{R}_{\sigma(i)}\right)\right)=p_{j}\left(\pi_{j}\left(R_{i}\right)\right)$ for each $j=1,2$ and $i=1, \ldots, k$.

From now we say that two $\omega$-limit sets are different if they are not equivalent (for an example of equivalent limit sets, see Figure 4). 


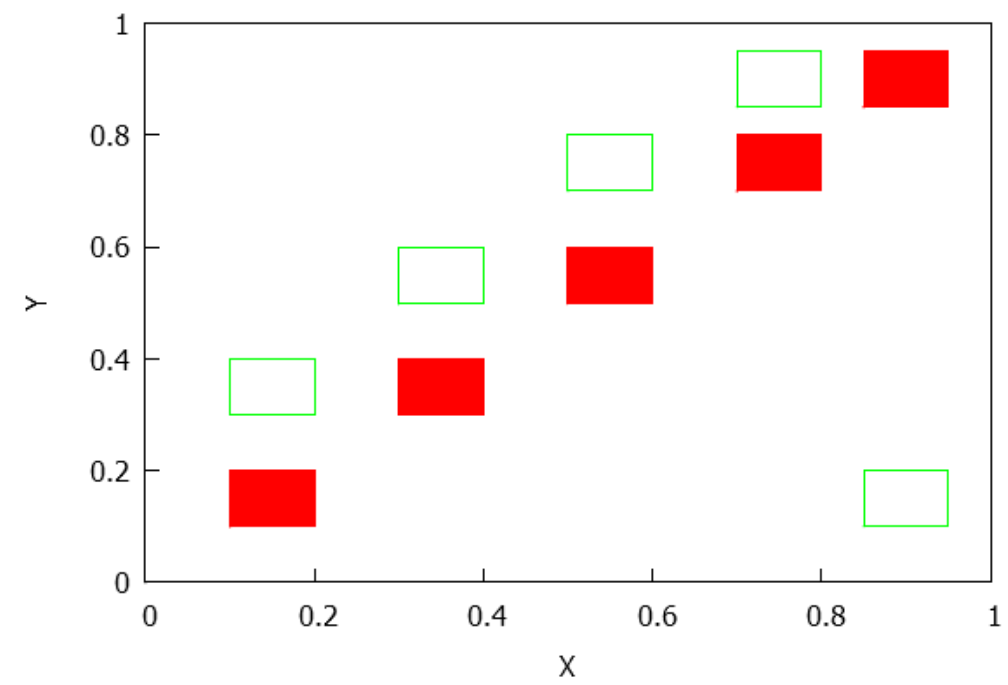

Figure 4. Two equivalent limit sets.

Finally, we recall the definition of the mixing $\omega$-limit set.

Definition 2. We say that the $\omega$-limit set $A$ is mixing if $\left.\left(f_{2} \circ f_{1}\right)^{p}\right|_{I_{i}}$ is mixing for all $i \in\{1, \ldots, p\}$. In other cases, we say that $A$ is no mixing.

In the next development, the strategy followed describing the $\omega$-limit sets with nonempty interiors consists in distinguishing the cases in which $A, B$ are or are not mixing.

\section{1. $A$ and $B$ Mixing}

Let $L=\omega_{F}(x, y)=\bigcup_{i=1}^{k} R_{i}$ be defined as before. Let $\ell \in \mathbb{N}$ be $\ell:=\operatorname{lcm}(p, q)$. Without loss of generality, rearranging the indexes if necessary, we can assume that

$$
\left(f_{2} \circ f_{1}\right)^{r}\left(I_{1}\right)=I_{1+r(\bmod p)} \text {, for } r=1, \ldots, p ; \quad\left(f_{1} \circ f_{2}\right)^{s}\left(J_{1}\right)=J_{1+s}(\bmod q) \text {, for } s=1, \ldots, q \text {. }
$$

We can distinguish two general subcases:

1. $f_{1}(A) \cap \operatorname{Int}(B) \neq \varnothing$ (or equivalently $f_{1}(A)=B$, Lemma 3). Therefore, $f_{1}(A)=B$ and $f_{2}(B)=A$, and there exists a bijective correspondence between the intervals of $A$ and $B$ and, hence, $p=q$. If $p=1$ the distribution is trivial, we only have a unique rectangle $R=I_{1} \times J_{1}$. For $p \geq 2$ we reason as follows. Assuming an ordering such that $R_{1}=I_{1} \times J_{1}$, then

$$
f_{1}\left(I_{1}\right)=J_{t}
$$

for some $t \in\{1, \ldots, p\}$. In particular, if $f_{1}\left(I_{1}\right)=J_{1}$ then $p_{2}\left(J_{1}\right) \geq 2$ since $p \geq 2$, although it is also possible to find examples for which $p_{2}\left(I_{1}\right)=1$. Observe that under the previous assumptions, according to (4) the condition $f_{1}\left(I_{1}\right)=J_{t}$ determines the images of all intervals $I_{i}$ and $J_{j}$ for $i, j=1, \ldots, p$. In fact, $f_{1}\left(I_{i}\right)=J_{i+t-1(\bmod p)}$ and $f_{2}\left(J_{i}\right)=I_{i+2-t}(\bmod p)$ for any $i \in\{1, \ldots p\}$. Thus, two different distributions (except equivalences) are possible:

(D1) If $t \neq 1$, there are $k=p=q$ rectangles and there exists an arrangement of the indexes such that $R_{i}=I_{i} \times J_{i}$ for $i=1, \ldots, k$. It is clear that $p_{1}\left(I_{i}\right)=p_{2}\left(J_{i}\right)=1$ for any $i=1, \ldots, k$. See Figure 5 .

(D2) If $t=1$, there are $k=2 p=2 q$ rectangles and $f_{1}\left(I_{i}\right)=J_{i(\bmod p)}$ and $f_{2}\left(J_{i}\right)=I_{i+1(\bmod p)}$, where $p \geq 2$. Also, it is held that $p_{1}\left(I_{i}\right)=\frac{k}{p}=2$ and $p_{2}\left(J_{i}\right)=\frac{k}{p}=2$ for $i=1, \ldots p$, see Figure 6 .

Observe that if the number of rectangles $k$ is odd, then only Distribution D1 is possible. 
2. $f_{1}(A) \cap \operatorname{Int}(B)=\varnothing$. We define

$$
B^{\prime}=\bigcup_{i=1}^{p} f_{1}\left(I_{i}\right)=\bigcup_{i=1}^{p} J_{i}^{\prime} \text { and } A^{\prime}=\bigcup_{i=1}^{q} f_{2}\left(J_{i}\right)=\bigcup_{i=1}^{q} I_{i}^{\prime} .
$$

Notice that $B^{\prime}=f_{1}(A)$ and $A^{\prime}=f_{2}(B)$. Moreover, $B^{\prime} \cap \operatorname{Int}(B)=\varnothing$ and $A^{\prime} \cap$ $\operatorname{Int}(A)=\varnothing$. In this case, the number of rectangles, $k$, is always even, in particular $k=2 \ell=2 \operatorname{lcm}(p, q)$ as $F^{2 r}\left(I_{1} \times J_{1}\right)=I_{1+r(\bmod p)} \times J_{1+r(\bmod q)}$ and $F^{2 r+1}\left(I_{1} \times J_{1}\right)=$ $f_{2}\left(J_{1+r(\bmod q)}\right) \times f_{1}\left(I_{1+r(\bmod p)}\right)=I_{1+r(\bmod q)}^{\prime} \times J_{1+r(\bmod p)}^{\prime}$ for $r \in\{1, \ldots, \ell\}$. The $\omega$-limit set $L$ is given by $L=L_{1} \cup L_{2}$, with $\pi_{1}\left(L_{1}\right)=A, \pi_{2}\left(L_{1}\right)=B, \pi_{1}\left(L_{2}\right)=A^{\prime}$, $\pi_{2}\left(L_{2}\right)=B^{\prime}$, such that $\operatorname{Int}\left(L_{1}\right) \cap L_{2}=\varnothing$. The number of rectangles $R_{i}$ of $L_{1}$ is $k_{1}$ and the number of rectangles $R_{i}^{\prime}$ of $L_{2}$ is $k_{2}$. Observe also that $k_{1}=k_{2}=\ell=\operatorname{lcm}(p, q)$, hence $k=2 \cdot k_{1}=2 \cdot \ell$ and $R_{i}=I_{i(\bmod p)} \times J_{i(\bmod q)}, R_{i}^{\prime}=I_{i(\bmod q)}^{\prime} \times J_{i(\bmod p)^{\prime}}^{\prime}$ $i \in\{1, \ldots, \ell\}$, with $p_{1}\left(\pi_{1}(R)\right)=\frac{\ell}{p}, p_{2}\left(\pi_{2}(R)\right)=\frac{\ell}{q}$ for each $R$ in $L_{1}$ and $p_{1}\left(\pi_{1}\left(R^{\prime}\right)\right)=$ $\frac{\ell}{q}, p_{2}\left(\pi_{2}\left(R^{\prime}\right)\right)=\frac{\ell}{p}$ for each $R^{\prime}$ in $L_{2}$. Thus, $p_{1}\left(I_{i}\right)=p_{2}\left(J_{i}^{\prime}\right)=\frac{\ell}{p}=\frac{k}{2 p}$ for $i \in\{1, \ldots, p\}$, and $p_{2}\left(J_{i}\right)=p_{1}\left(I_{i}^{\prime}\right)=\frac{\ell}{q}=\frac{k}{2 q}$ for $i \in\{1, \ldots, q\}$. As we have signaled above, the rectangles of the $\omega$-limit set are described by $R_{i(\bmod k)}=I_{i(\bmod p)} \times J_{i(\bmod q)}$ and $R_{i(\bmod k)}^{\prime}=I_{i(\bmod q)}^{\prime} \times J_{i(\bmod p)}^{\prime}$ for $i=1, \ldots, k$. This case is easily detected when there exists $i \neq j$ such that $p_{1}\left(\pi_{1}\left(R_{i}\right)\right)=p_{2}\left(\pi_{2}\left(R_{j}^{\prime}\right)\right) \neq p_{1}\left(\pi_{1}\left(R_{j}^{\prime}\right)\right)=p_{2}\left(\pi_{2}\left(R_{i}\right)\right)$. Additionally, if:

(D3) $f_{1}(A) \cap B=\varnothing$. There are $2 \ell$ connected components, since the number of connected components of $A \cup A^{\prime}$ is $p+q$. See Figure 7 .

(D4) $f_{1}(A) \cap B \neq \varnothing$. The number of connected components depends on the number of connected components of $A \cup A^{\prime}$ and $B \cup B^{\prime}$ which is less than or equal to $p$ and $q$, respectively, see Lemma 4 . Consequently, rectangles can be disjoint, have a common vertex or two common vertices, see Figures 8-11. Notice that the case for two common vertices can only be realized by a unique distribution, given precisely by Figure 10; moreover, observe that $R_{i} \cap R_{j}^{\prime} \neq \varnothing$ if and only if $I_{i} \cap I_{j}^{\prime} \neq \varnothing$ and $J_{i} \cap J_{j}^{\prime} \neq \varnothing$.

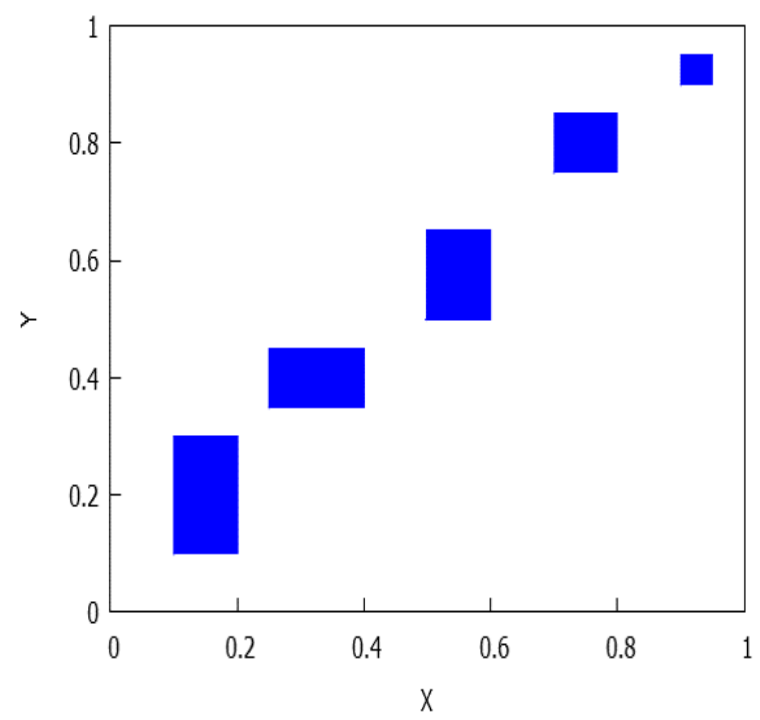

Figure 5. Mixing-mixing. Distribution D1. $k=5 ; f_{1}(A)=B, A$ and $B$ contain 5 disjoint intervals, that is, $p=q=5 ; k=\ell=\operatorname{lcm}(5,5)=5$. Observe that $p_{1}(I)=1$ and $p_{2}(J)=1$ for each rectangle $R=I \times J$. 

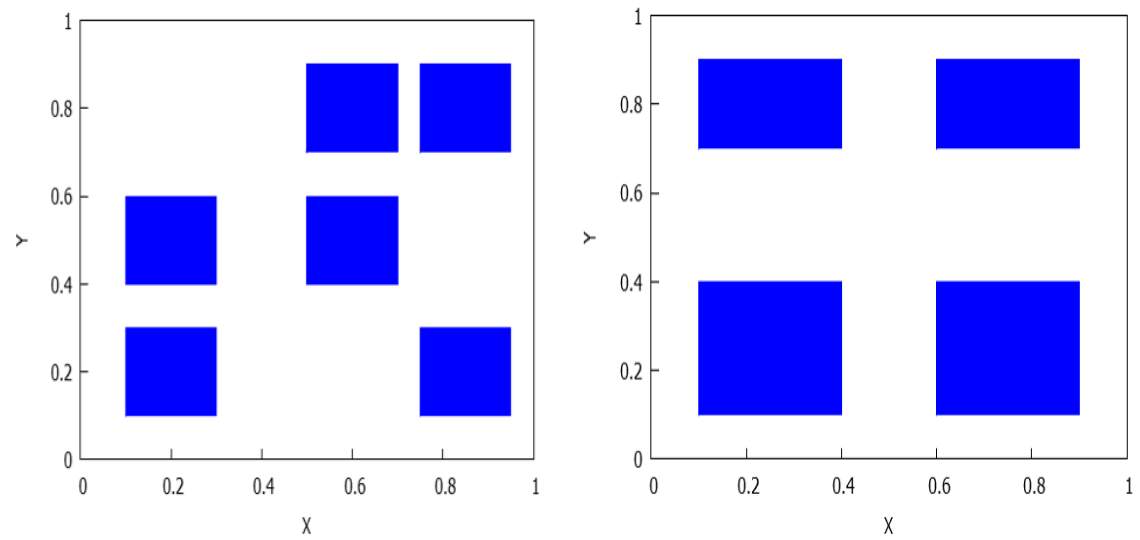

Figure 6. Mixing-mixing. Distribution D2. $f_{1}(A) \cap \operatorname{Int}(B) \neq \varnothing$. In the left side: $p=q=3, \ell=3$ and $k=2 \cdot \ell=6 ; p_{1}\left(\pi_{1}(R)\right)=2$ and $p_{2}\left(\pi_{2}(R)\right)=2$ for each rectangle $R$ in $L$. In the right side: $p=q=2, \ell=2, k=2 \ell=4$; again $p_{1}\left(\pi_{1}(R)\right)=2$ and $p_{2}\left(\pi_{2}(R)\right)=2$.

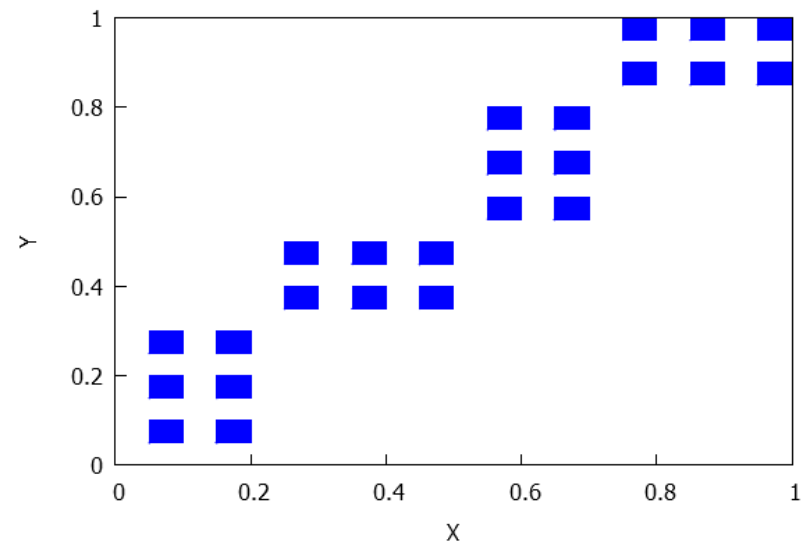

Figure 7. $A$ and $B$ mixing. Distribution D3. We define $L_{1}$ as the set of rectangles $R$ such that $p_{1}\left(\pi_{1}(R)\right)=3$ and $p_{2}\left(\pi_{2}(R)\right)=2$. The contrary occurs for rectangles $R^{\prime}$ in $L_{2}$, for which $p_{1}\left(R^{\prime}\right)=2$ and $p_{2}\left(R^{\prime}\right)=3$. Thus, $\left|L_{1}\right|=\left|L_{2}\right|=12$ and $|L|=\left|L_{1} \cup L_{2}\right|=24 ; A$ contains 4 disjoint intervals, $B$ is made of 6 disjoint intervals and $f_{1}(A) \cap B=\varnothing$. Observe that $\ell=\operatorname{lcm}(4,6)=12$.

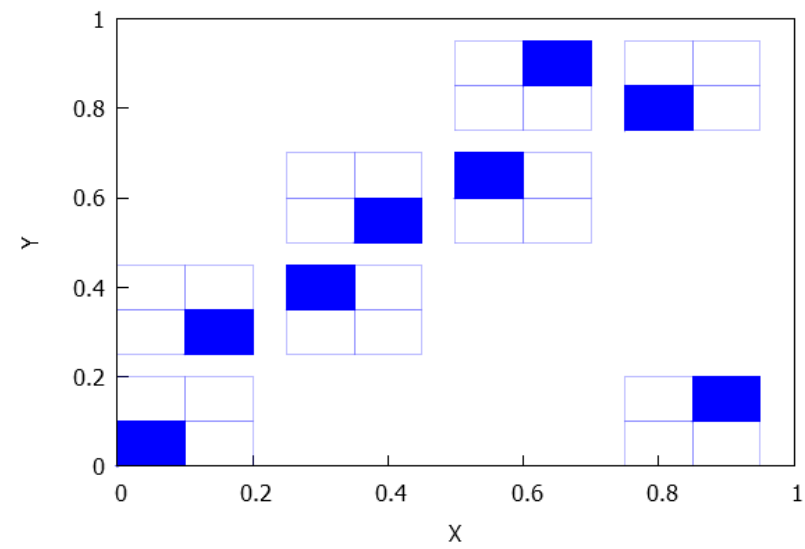

Figure 8. $A$ and $B$ mixing. Distribution D4. $|L|=8$. Observe that $p_{i}\left(\pi_{i}(R)\right)=1$ for any $R$ in $L$ and $i=1$, 2. Thus, $\left|L_{1}\right|=4$ and $\left|L_{2}\right|=4$. The cardinality of $A$ and $B$ is equal to 4 , thus $\ell=4$; $f_{1}(A) \cap \operatorname{Int}(B)=\varnothing$ and $f_{1}(A) \cap B \neq \varnothing$, for this reason projections of rectangles have common vertices. Observe that rectangles do not have common points. 


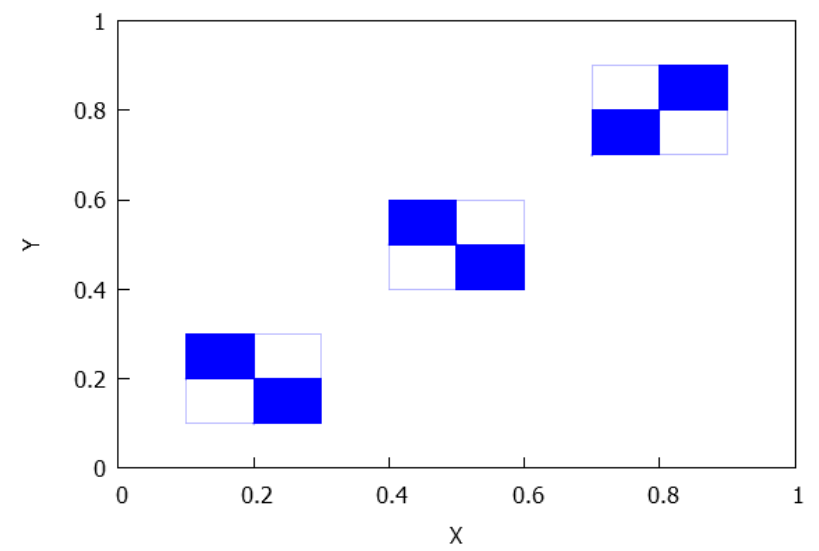

Figure 9. $A$ and $B$ mixing. Distribution D4. $|L|=6$. $A$ contains 3 disjoint intervals and $f_{1}(A) \cap$ $\operatorname{Int}(B)=\varnothing$ but $f_{1}(A) \cap B \neq \varnothing ; B$ has 3 disjoint intervals. $\left|L_{1}\right|=\left|L_{2}\right|=3$ and rectangles of both sets have a common vertex.

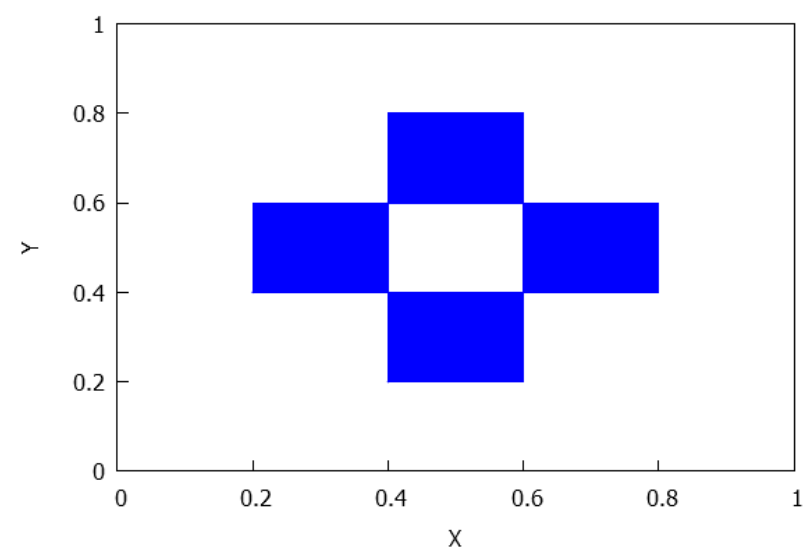

Figure 10. $A$ and $B$ mixing. Distribution D4. $|L|=4$. $A$ contains 1 interval and $f_{1}(A) \cap \operatorname{Int}(B)=\varnothing$ but $f_{1}(A) \cap B \neq \varnothing$; in this case, the intersection are two points; $B$ has 2 intervals; $\left|L_{1}\right|=\left|L_{2}\right|=2$. Rectangles from $L_{1}$ have two vertices in common with rectangles from $L_{2}$ and the same for rectangles from $L_{2}$.

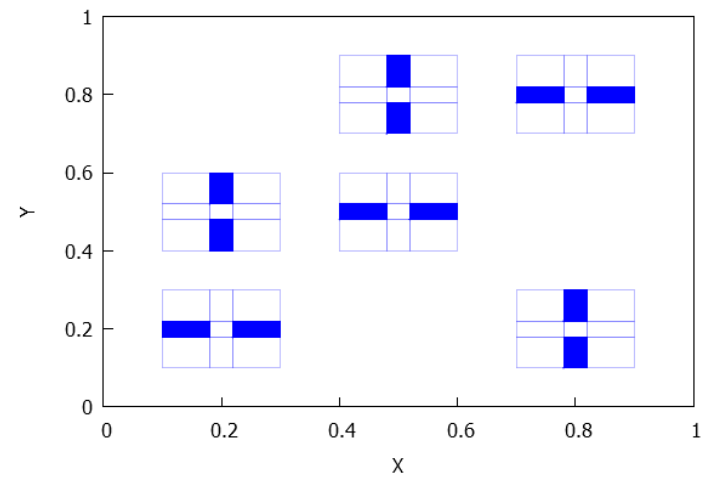

Figure 11. $A$ and $B$ mixing. Distribution D4. $|L|=12 ; A$ contains 3 intervals and $f_{1}(A) \cap \operatorname{Int}(B)=\varnothing$ but $f_{1}(A) \cap B \neq \varnothing$; in this case, the intersection are two points but rectangles have no common points; $B$ has 6 intervals; $\left|L_{1}\right|=\left|L_{2}\right|=6$.

\subsection{A Mixing and B No Mixing}

Since $B$ is no mixing and by Definition 2, Lemma 1 and Lemma 2, there are closed intervals $J_{i}^{1}$ and $J_{i}^{2}$ whose intersection is a fixed point of $\left(f_{1} \circ f_{2}\right)^{q}$ and such that $J_{i}=J_{i}^{1} \cup J_{i}^{2}$, $\left(f_{1} \circ f_{2}\right)^{q}\left(J_{i}^{1}\right)=J_{i}^{2},\left(f_{1} \circ f_{2}\right)^{q}\left(J_{i}^{2}\right)=J_{i}^{1}$, and $\left.\left(f_{1} \circ f_{2}\right)^{2 q}\right|_{J_{i}^{j}}$ is mixing on $J_{i}^{j}$ for $i=1,2, \ldots, q$ 
and $j=1,2$. Now, it is not possible that $f_{1}(A) \cap \operatorname{Int}(B) \neq \varnothing$ due to Lemma 3 and the different mixing character of the intervals.

Assume that $L=L_{1} \cup L_{2}$ and that the rectangles of $L_{1}$ and $L_{2}$ are of type $I_{i} \times J_{j}^{r}$ and type $\left(I^{\prime}\right)_{j}^{r} \times J_{i}^{\prime}$ (with $i \in\{1, \ldots, p\}, j \in\{1, \ldots, q\}, r=1,2$ ), respectively, where we have implicitly used Proposition 1 as well as the notation

$$
f_{1}(A)=f_{1}\left(\bigcup_{i=1}^{p} I_{i}\right)=\bigcup_{i=1}^{p} f_{1}\left(I_{i}\right)=: \bigcup_{i=1}^{p} J_{i}^{\prime}
$$

and

$$
f_{2}(B)=f_{2}\left(\bigcup_{i=1}^{q}\left(J_{j}^{1} \cup J_{j}^{2}\right)\right)=\bigcup_{j=1}^{q}\left(f_{2}\left(J_{j}^{1}\right) \cup f_{2}\left(J_{j}^{2}\right)\right)=: \bigcup_{j=1}^{q}\left(\left(I^{\prime}\right)_{j}^{1} \cup\left(I^{\prime}\right)_{j}^{2}\right) .
$$

Next, by assuming that $R_{1}=I_{1} \times J_{1}^{1}$, we have:

- If $\frac{\ell}{q}$ is odd, $\ell=r q, r$ odd, then $\left(f_{1} \circ f_{2}\right)^{r q}\left(J_{j}^{s}\right)=J_{j}^{t}$, for all $j=1, \ldots, q$ and $s, t \in\{1,2\}$, $s \neq t$, due to the fact that $B$ is not mixing; then both rectangles $I_{i} \times J_{j}^{1}$ and $I_{i} \times J_{j}^{2}$ belong to $L_{1}$ (realize that $\left.F^{2 \ell}\left(I_{i} \times J_{j}^{1}\right)=\left(f_{2} \circ f_{1}\right)^{\ell}\left(I_{i}\right) \times\left(f_{1} \circ f_{2}\right)^{\ell}\left(J_{j}^{1}\right)=I_{i} \times J_{j}^{2}\right)$, that is, $I_{i} \times J_{j}$ belongs to $L_{1}$; similarly, both rectangles $\left(I^{\prime}\right)_{j}^{1} \times J_{i}$ and $\left(I^{\prime}\right)_{j}^{2} \times J_{i}$ belong to $L_{2}$ and, therefore, the distribution of $L=L_{1} \cup L_{2}$ is reduced to the case $A$ and $B$ mixing with $f_{1}(A) \cap B=\varnothing$ (distribution D3).

- If $\frac{\ell}{q}$ is even, $\ell=m q, m$ even, now each rectangle of the $\omega$-limit $L$ is the half part of a rectangle $I_{i} \times J_{k}$ or $I_{j}^{\prime} \times J_{i}^{\prime}$, and the number of rectangles of $L_{n}$ is equal to $\ell, n=1,2$, so $|L|=2 \ell$ (notice that, as a consequence of the parity of $\ell, F^{2 \ell}\left(I_{1} \times J_{1}^{1}\right)=\left(f_{2} \circ\right.$ $\left.f_{1}\right)^{\ell}\left(I_{1}\right) \times\left(f_{1} \circ f_{2}\right)^{\ell}\left(J_{1}^{1}\right)=I_{1} \times J_{1}^{1}$ and consider that $F^{t}\left(I_{1} \times J_{1}^{1}\right) \notin\left\{I_{1} \times J_{1}^{1}, I_{1} \times J_{2}^{1}\right\}$ for $0<t<2 \ell$.

Thus, we assume that $\frac{\ell}{q}$ is even. The number of rectangles of $L$ must be a multiple of 4. Since $L_{1}$ has an even number of rectangles, the following cases sum up the possibilities.

(D5) $f_{1}(A) \cap B=\varnothing$. In this case, the $\omega$-limit is a set $L$ which is the union of $L_{1}$ and $L_{2}$ both with the same number of rectangles. We also have that $L_{i}$ has an even number of rectangles for $i=1,2$. Observe that: $p_{1}\left(\pi_{1}(R)\right)=\frac{\ell}{p}$ and $p_{2}\left(\pi_{2}(R)\right)=\frac{\ell}{q}$ for each entire rectangle $R$ having the form $I_{i} \times J_{j}=I_{i} \times\left(J_{j}^{1} \cup J_{j}^{2}\right)$ and each projection of a rectangle of $L_{1}$ must have a common point with the projection of another rectangle; similarly, $p_{2}\left(\pi_{2}(R)\right)=\frac{\ell}{p}$ and $p_{1}\left(\pi_{1}(R)\right)=\frac{\ell}{q}$ for each entire rectangle $R \in L_{2}$ (having the form $\left.\left(I^{\prime}\right)_{j} \times\left(J^{\prime}\right)_{i}=\left(\left(I^{\prime}\right)_{j}^{1} \cup\left(I^{\prime}\right)_{j}^{2}\right) \times\left(\left(J^{\prime}\right)_{i}\right)\right)$ and each projection of a rectangle must have a common point with the projection of another rectangle. See Figure 12.

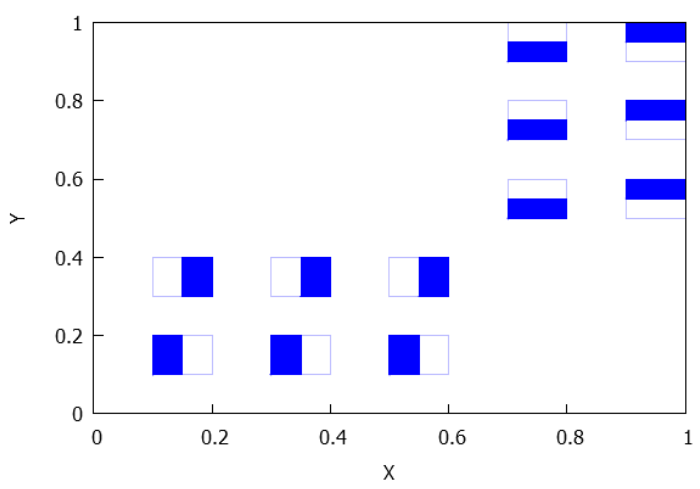

Figure 12. $A$ mixing and $B$ no mixing. Distribution D5. Here, $p=2, q=3, \ell=6$; each 'entire' rectangle $R$ in $L_{1}$ verifies $p_{1}\left(\pi_{1}(R)\right)=3=\frac{\ell}{p}$ and $p_{2}\left(\pi_{2}(R)\right)=2=\frac{\ell}{q}$, whereas each 'entire' rectangle $R^{\prime}$ in $L_{2}$ holds $p_{2}\left(\pi_{2}\left(R^{\prime}\right)\right)=3=\frac{\ell}{p}$ and $p_{1}\left(\pi_{1}\left(R^{\prime}\right)\right)=2=\frac{\ell}{q}$. 
(D6) $f_{1}(A) \cap \operatorname{Int}(B)=\varnothing$ and $f_{1}(A) \cap B \neq \varnothing$. By Lemma 4 and Remark 2 , we know that each connected component of $A \cup f_{2}(B)$ has the form $I_{i} \cup I_{i}^{\prime} \cup I_{i+1}$, where $I_{i}=\left[a_{i}, b_{i}\right]$, $I_{i}^{\prime}=\left[b_{i}, a_{i+i}\right], I_{i+1}=\left[a_{i+1}, b_{i+1}\right]$, for some values $a_{i}<b_{i}<a_{i+1}<b_{i+1}$. Notice that $p=2 q, \ell=p, \frac{\ell}{q}=2$; moreover, the number of rectangles in $L=L_{1} \cup L_{2}$ is just $4 q$. Here, the 'entire' rectangles $R$ in $L_{1}$ have the form $I_{i} \times J_{j}=I_{i} \times\left(J_{j}^{1} \cup J_{j}^{2}\right)$, and we have $p_{1}\left(\pi_{1}(R)\right)=\frac{\ell}{p}=1$ and $p_{2}\left(\pi_{2}(R)\right)=\frac{\ell}{q}=2$. For rectangles $R^{\prime}$ in $L_{2}$, having type $\left(\left(I^{\prime}\right)_{i}^{1} \cup\left(I^{\prime}\right)_{i}^{2}\right) \times J_{j}^{\prime}$, instead we find $p_{1}\left(\pi_{1}\left(R^{\prime}\right)\right)=\frac{\ell}{q}=2$ and $p_{2}\left(\pi_{2}(R)\right)=\frac{\ell}{p}=1$. See Figure 13.
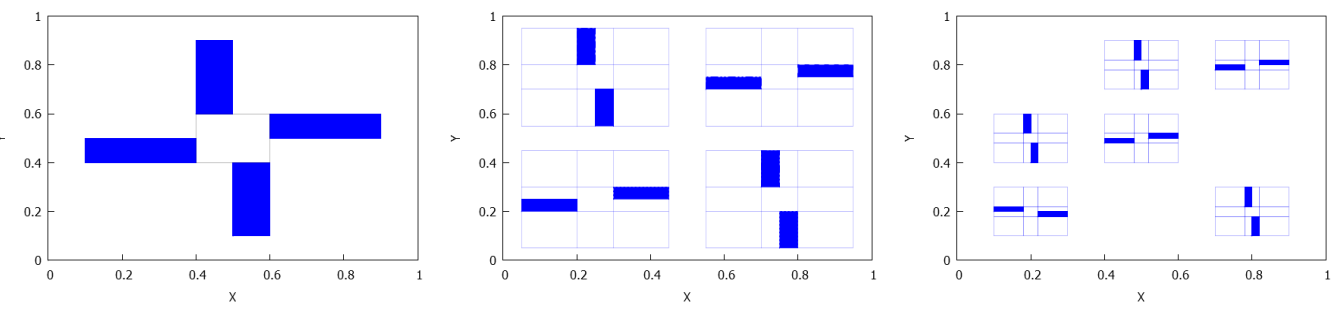

Figure 13. $A$ mixing and $B$ no mixing. Distribution D6. In the first examples, $p=2, q=1$, and $p=4, q=2$; in the last one, $p=6, q=3$.

\section{3. $A$ and $B$ No Mixing}

Realize that if $A$ and $B$ are no mixing, then $f_{1}(A)$ and $f_{2}(B)$ are also no mixing. We can assume that, except for equivalences, there are closed intervals $I_{i}^{1}$ and $I_{i}^{2}$, whose intersection is a fixed point of $\left(f_{2} \circ f_{1}\right)^{p}$ and such that $I_{i}=I_{i}^{1} \cup I_{i}^{2},\left(f_{2} \circ f_{1}\right)^{p}\left(I_{i}^{1}\right)=I_{i}^{2},\left(f_{2} \circ f_{1}\right)^{p}\left(I_{i}^{2}\right)=I_{i}^{1}$ and $\left.\left(f_{2} \circ f_{1}\right)^{2 p}\right|_{I_{i}^{j}}$ is mixing on $I_{i}^{j}$ for $i=1,2, \ldots, p$ and $j=1,2$. A similar situation occurs for $B$, with $J_{j}=J_{j}^{1} \cup J_{j}^{2}, j=1, \ldots, q$. We also assume that $R_{1}=I_{1}^{1} \times J_{1}^{1}$. Let us assume also that $I_{i}^{1}<I_{i}^{2}$ and $J_{i}^{1}<J_{i}^{2}$, where $I<J$ means that $x<y$ for each $x \in I$ and $y \in J$. Now, according to Lemma 4 , only the cases $f_{1}(A) \cap B=\varnothing$ and $f_{1}(A) \cap \operatorname{Int}(B) \neq \varnothing$ are admissible (notice that the last one is equivalent to $f_{1}(A)=B$ ). Recall that $\ell=\operatorname{lcm}(p, q)$, $p$ and $q$ being the number of intervals in $A$ and $B$, respectively. Moreover, thanks to the equivalence relation given in Definition 1 , we can assume without loss of generality that $\left(f_{2} \circ f_{1}\right)\left(I_{i}\right)=I_{i+1}(\bmod p)$ and $\left(f_{1} \circ f_{2}\right)\left(J_{j}\right)=J_{j+1}(\bmod q)$.

1. Case $f_{1}(A) \cap B=\varnothing$. Firstly, we claim that the number of rectangles in the orbit of $I_{1} \times J_{1}, \operatorname{Orb}_{F}\left(I_{1} \times J_{1}\right)=\left\{I_{1} \times J_{1}, f_{2}\left(J_{1}\right) \times f_{1}\left(I_{1}\right),\left(f_{2} \circ f_{1}\right)\left(I_{1}\right) \times\left(f_{1} \circ f_{2}\right)\left(J_{1}\right), \ldots\right\}$, is equal to $2 \ell$. To see it, let $\{a\}=I_{1}^{1} \cap I_{1}^{2},\{b\}=J_{1}^{1} \cap J_{1}^{2}$; we know that $a(b)$ is a periodic point of $f_{2} \circ f_{1}\left(f_{1} \circ f_{2}\right)$ of period $p(q)$. We assert that $(a, b)$ is a periodic point of $F$ of period $2 \ell$. Let $m$ be the order of $(a, b)$ for the map $F$. Since $F^{2 \ell}(a, b)=$ $\left(\left(f_{2} \circ f_{1}\right)^{\ell}(a),\left(f_{1} \circ f_{2}\right)^{\ell}(b)\right)=(a, b)$, on the one hand we have $m \mid 2 \ell$. Thus, $F^{2 m}(a, b)=$ $\left(\left(f_{2} \circ f_{1}\right)^{m}(a),\left(f_{1} \circ f_{2}\right)^{m}(b)\right)=(a, b)$; on the other hand, we also deduce that $p \mid m$ and $q \mid m$, and consequently $\ell \mid m$. From the above reasoning, either $m=\ell$ or $m=2 \ell$. If it were $m=\ell$, with $\ell$ even, $\ell=2\left(\frac{\ell}{2}\right)$, then $F^{\ell}(a, b)=\left(\left(f_{2} \circ f_{1}\right)^{\frac{\ell}{2}}(a),\left(f_{1} \circ f_{2}\right)^{\frac{\ell}{2}}(b)\right)=$ $(a, b)$ and $p\left|\frac{\ell}{2}, q\right| \frac{\ell}{2}$, in contradiction with the definition of the lowest common multiple. If it were $m=\ell$, with $\ell$ odd, $\ell=2 s+1$, now $F^{2 s+1}(a, b)=(a, b)$ would give us $\left(f_{2} \circ f_{1}\right)^{S}\left(f_{2}(b)\right)=a,\left(f_{1} \circ f_{2}\right)^{S}\left(f_{1}(a)\right)=b$, so $(a, b) \in(A \times B) \cap\left(f_{2}(B) \times f_{1}(A)\right)$, that is, $a \in A \cap f_{2}(B)$, contrary to our hypothesis. Therefore, $(a, b)$ is $F$-periodic and its period is $2 \ell$. As a consequence, $\operatorname{Orb}_{F}\left(I_{1} \times J_{1}\right)$ has $2 \ell$ different rectangles (maybe with common vertexes). Since $F^{2 \ell}\left(I_{1} \times J_{1}\right)=I_{1} \times J_{1}$, and $\ell$ is the first time $s$ in which $\left(f_{2} \circ f_{1}\right)^{S}\left(I_{1}\right) \times\left(f_{1} \circ f_{2}\right)^{S}\left(J_{1}\right)=I_{1} \times J_{1}$, we have that $\operatorname{Orb}_{F}\left(I_{1} \times J_{1}\right)$ has either $\ell$ or $2 \ell$ elements. But the case $\ell$ is forbidden because then $F^{\ell}(a, b)=(a, b)$ due to the fact that $(a, b), F(a, b), \ldots$, are the 'middle points' of $I_{1} \times J_{1}$ and its respective images. This ends the claim. 
The last claim does not imply necessarily that the number $k$ of rectangles in the $\omega$-limit $L=\omega_{F}(x, y)=\operatorname{Orb}_{F}\left(I_{1}^{1} \times J_{1}^{1}\right)$ must be $2 \ell$. It will depend on the parity of $k_{1}:=\frac{\ell}{p}$ and $k_{2}:=\frac{\ell}{q}$. Realize that $k_{1}$ and $k_{2}$ cannot be even simultaneously, at least some of them are odd.

If $k_{1}$ is odd and $k_{2}$ is even (the case $k_{1}$ even and $k_{2}$ odd is analogous), then $F^{2 \ell}\left(I_{1}^{1} \times\right.$ $\left.J_{1}^{1}\right)=\left(f_{2} \circ f_{1}\right)^{\ell}\left(I_{1}^{1}\right) \times\left(f_{1} \circ f_{2}\right)^{\ell}\left(J_{1}^{1}\right)=I_{1}^{2} \times J_{1}^{1}$, and really we have $2 \ell$ different rectangles in $L$ because the parts $I_{1}^{1} \times J_{1}^{1}$ and $I_{1}^{2} \times J_{1}^{1}$ gives rise to the union of $I_{1} \times J_{1}^{1}$. The distribution that appears is equal to the case mixing-no mixing previously considered, distribution D5.

If $k_{1}$ and $k_{2}$ are both odd integers, then $\ell=p k_{1}=q k_{2}$ and $F^{2 \ell}\left(I_{1}^{1} \times J_{1}^{1}\right)=\left(f_{2} \circ\right.$ $\left.f_{1}\right)^{\ell}\left(I_{1}^{1}\right) \times\left(f_{1} \circ f_{2}\right)^{\ell}\left(J_{1}^{1}\right)=I_{1}^{2} \times J_{1}^{2}$, and in this case we find $4 \ell$ different rectangles (notice that $F^{2 j}\left(I_{1}^{1} \times J_{1}^{1}\right) \neq I_{1}^{1} \times J_{1}^{1}$ for $j=1, \ldots, \ell-1$ ); moreover, the rectangles in the $\omega$-limit $L$ are distributed in two groups of rectangles, namely $L_{1}$ and $L_{2}$, with $\left|L_{1}\right|=\left|L_{2}\right|=2 \ell$. The distribution of $L_{2}$ mimics the distribution of $L_{1}$, shifting the position of the axes. See Figure 14.

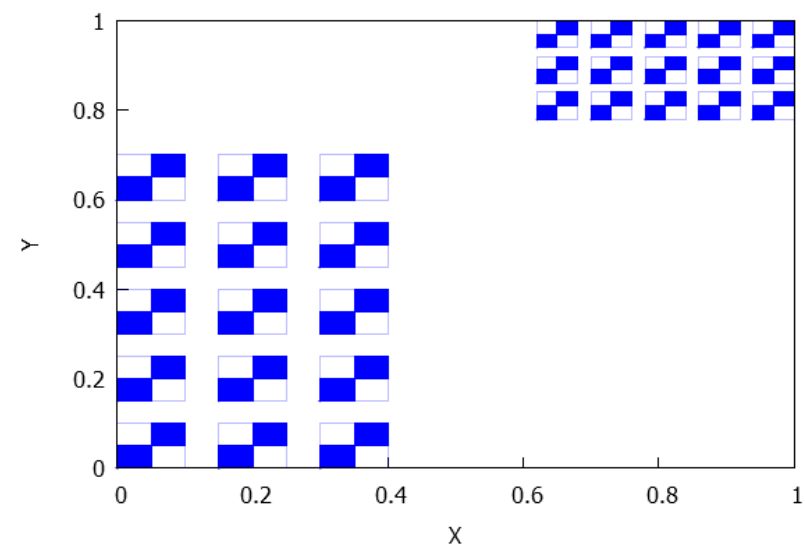

Figure 14. $A$ and $B$ no mixing. Observe that $f(A) \cap B=\varnothing$ and under the theoretical assumptions we should obtain 7 (2 times in each) changes of sign, but shifting $J_{2}, J_{4}$ and $I_{2}$ the result is as it is shown.

Thus, let us assume $k_{1}$ and $k_{2}$ are odd numbers. Then, structures that are made up of two rectangles with a common point appear, which allows us to consider different orientations, namely, $(+)$ and $(-)$ see Figure 15.
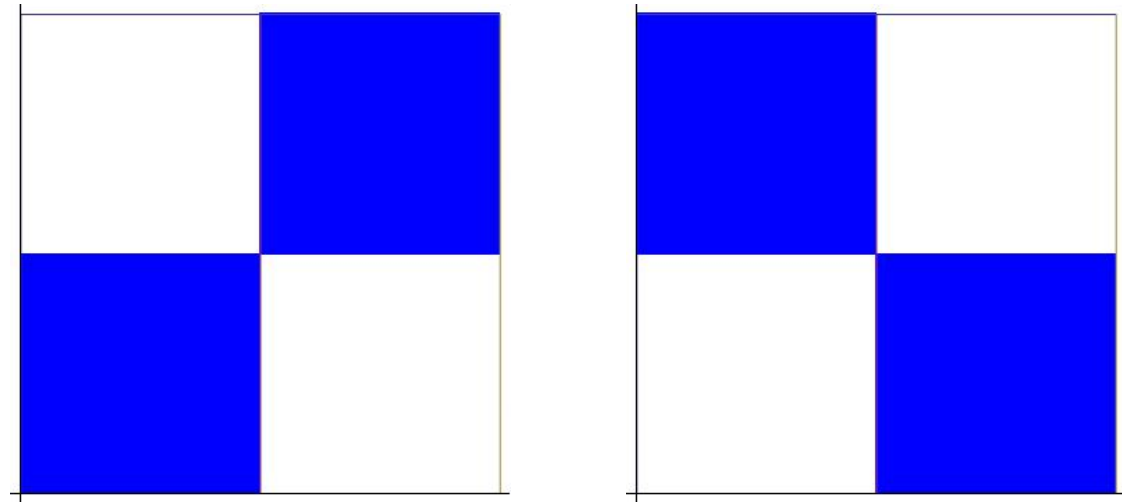

Figure 15. Distributions with two different orientations for the case no mixing-no mixing. Positive orientation is shown in the left and negative one is in the right.

For this case, there must coexist these types of structures with both orientations, but, how many of them?

In order to describe and count the structures with negative orientation, we proceed as follows. Recall that we assume $A=\cup_{i=1}^{p} I_{i}, B=\cup_{j=1}^{q} J_{j}$, and both $\left.\left(f_{2} \circ f_{1}\right)^{p}\right|_{I_{i}}$ and 
$\left.\left(f_{1} \circ f_{2}\right)^{q}\right|_{J_{j}}$ are no mixing. We put $I_{i}=I_{i}^{1} \cup I_{i}^{2}, J_{j}=J_{j}^{1} \cup J_{j}^{2}$, with $I_{i}^{1} \leq I_{i}^{2}, J_{j}^{1} \leq J_{j}^{2}$. Moreover, $p \geq q, \ell=\operatorname{lcm}(p, q)$ and $k_{1}=\frac{\ell}{p}, k_{2}=\frac{\ell}{q}$ are odd integers. If the $\omega$-limit set $L$ is written as $L=L_{1} \cup L_{2}$, take into account that we are going to describe the structures with negative orientation in $L_{1}$, for subrectangles contained in bigger rectangles of type $I_{i} \times J_{j}$, and that we start to iterate with the subrectangle $I_{1}^{1} \times J_{1}^{1}$. For $i=1, \ldots, p$ define $\sigma(j) \in\{1,2\}$ as the value for which

$$
\left(f_{2} \circ f_{1}\right)^{i}\left(I_{1}^{1}\right)=I_{1+i(\bmod (p))}^{\sigma(i)} .
$$

Notice that $\sigma(p)=2$.

Similarly, for $j=1, \ldots, q$, define $\tau(j) \in\{1,2\}$ to be value holding

$$
\left(f_{1} \circ f_{2}\right)^{j}\left(J_{1}^{1}\right)=J_{1+j(\bmod (q))}^{\tau(j)} .
$$

From the mixing property of $f_{1} \circ f_{2}$, we have $\tau(q)=2$. In short,

$$
\left(\begin{array}{ccccc}
1 & 2 & \ldots & p-1 & p \\
\sigma(1) & \sigma(2) & \ldots & \sigma(p-1) & 2
\end{array}\right),\left(\begin{array}{ccccc}
1 & 2 & \ldots & q-1 & q \\
\tau(1) & \tau(2) & \ldots & \tau(q-1) & 2
\end{array}\right) .
$$

We can extend the definition to two sequences

$$
\begin{gathered}
\sigma: \mathbb{N} \rightarrow\{1,2\}^{\mathbb{N}}, \\
\tau: \mathbb{N} \rightarrow\{1,2\}^{\mathbb{N}},
\end{gathered}
$$

in this manner: from the mixing properties of $f_{2} \circ f_{1}$ and $f_{1} \circ f_{2}$, we know, for instance, that $\left(f_{2} \circ f_{1}\right)^{p+1}\left(I_{1}^{1}\right)=\left(f_{2} \circ f_{1}\right)\left(I_{1}^{2}\right)=I_{1}^{\overline{\sigma(1)}}$, where we use the notation $\bar{r}$ to denote the value

$$
\bar{r}=1+r(\bmod (2)) \in\{1,2\} ;
$$

therefore, we can define $\sigma(p+j)=\overline{\sigma(j)}$ for $j=1, \ldots, p$. Similarly, $\left(f_{2} \circ f_{1}\right)^{2 p+1}\left(I_{1}^{1}\right)=$ $\left(f_{2} \circ f_{1}\right)\left(I_{1}^{1}\right)=I_{1}^{\sigma(1)}$, and we can define $\sigma(2 p+j)=\sigma(j)$ for $j=1, \ldots, p$. Thus,

$$
\sigma(n)= \begin{cases}\sigma(r), & \text { if } n=(2 j) p+r, \text { for some } j \geq 0, r \in\{1, \ldots, p\}, \\ \overline{\sigma(r)}, & \text { if } n=(2 j+1) p+r, \text { for some } j \geq 0, r \in\{1, \ldots, p\} .\end{cases}
$$

Notice that $\left(f_{2} \circ f_{1}\right)^{n}\left(I_{1}^{1}\right)=I_{1+n(\bmod (p))}^{\sigma(n)}$. Similarly, we extend $\tau$ as

$$
\tau(n)=\left\{\begin{array}{l}
\sigma(s), \quad \text { if } n=(2 j) q+s, \text { for some } j \geq 0, s \in\{1, \ldots, q\}, \\
\overline{\sigma(s),} \quad \text { if } n=(2 j+1) q+s, \text { for some } j \geq 0, s \in\{1, \ldots, q\} .
\end{array}\right.
$$

Here, $\left(f_{1} \circ f_{2}\right)^{n}\left(I_{1}^{1}\right)=I_{1+n(\bmod (q))}^{\tau(n)}$.

Notice that $F^{2 \ell}\left(I_{1}^{1} \times J_{1}^{1}\right)=\left(f_{2} \circ f_{1}\right)^{\ell}\left(I_{1}^{1}\right) \times\left(f_{1} \circ f_{2}\right)^{\ell}\left(J_{1}^{1}\right)=I_{1}^{\sigma(\ell)} \times J_{1}^{\tau(\ell)}$, and the same time $F^{2 \ell}\left(I_{1}^{1} \times J_{1}^{1}\right)=\left(\left(f_{2} \circ f_{1}\right)^{p}\right)^{k_{1}}\left(I_{1}^{1}\right) \times\left(\left(f_{1} \circ f_{2}\right)^{q}\right)^{k_{2}}\left(J_{1}^{1}\right)=I_{1}^{2} \times J_{1}^{2}$. This implies that $\sigma(\ell)=\tau(\ell)=2$ because $k_{1}$ and $k_{2}$ are odd integers, and we can observe that from this point the values of the orbit of $I_{1}^{1} \times J_{1}^{1}$ belong to the same structure.

Notice also that if $i \in\{1, \ldots, \ell\}$ then

$$
F^{2(\ell+i)}\left(I_{1}^{1} \times J_{1}^{1}\right)=F^{2 i}\left(I_{1}^{2} \times J_{1}^{2}\right)=I_{1+i(\bmod (p))}^{\overline{\sigma(i)}} \times J_{1+i(\bmod (q))^{\prime}}^{\overline{\tau(i)}}
$$

whenever $F^{2 i}\left(I_{1}^{1} \times J_{1}^{1}\right)=I_{1+i(\bmod (p))}^{\sigma(i)} \times J_{1+i(\bmod (q))}^{\tau(i)}$. As we see, this again guarantees that our extensions, $\sigma, \tau: \mathbb{N} \rightarrow\{1,2\}^{\mathbb{N}}$ are well defined. 
Additionally, in order to check if the structure has positive or negative orientation, it suffices, according to (5), to analyze the pairs $(\sigma(i), \tau(i))$, with $i \in\{1, \ldots, \ell\}$. To this regard, define

$$
\mathrm{O}(i)=(\sigma(i), \tau(i)), i=1, \ldots, \ell .
$$

If $\mathrm{O}(i)=(r, r)$ for some $r \in\{1,2\}$, we say that $I_{1+i(\bmod (p))}^{\sigma(i)} \times J_{1+i(\bmod (q))}^{\tau(i)}$ has positive orientation; if, on the contrary, $\mathrm{O}(i)=(r, \bar{r})$, for $r \in\{1,2\}$, we say that the structure has negative orientation.

Consequently, if we try to count the positive and negative orientations, we only need to see the character of the pairs $\mathrm{O}(i)=(\sigma(i), \tau(i)), i=1, \ldots, \ell$.

For instance, if $p=5$ and $q=3$, with

$$
\left(\begin{array}{lllll}
1 & 2 & 3 & 4 & 5 \\
1 & 2 & 2 & 1 & 2
\end{array}\right),\left(\begin{array}{lll}
1 & 2 & 3 \\
2 & 1 & 2
\end{array}\right)
$$

we obtain:

$$
\begin{aligned}
& (1,1) \quad \rightarrow \quad(\sigma(1), \tau(1))=(1,2) \quad \rightarrow \quad(\sigma(2), \tau(2))=(2,1) \quad \rightarrow \\
& (\underline{\sigma(3)}, \underline{\tau(3))}=(2,2) \quad \rightarrow \quad(\underline{\sigma(4)}, \overline{\tau(1)})=(1,1) \quad \rightarrow \quad(\sigma(5), \overline{\tau(2)})=(2,2) \quad \rightarrow \\
& (\overline{\sigma(1)}, \overline{\tau(3)})=(2,1) \quad \rightarrow \quad(\overline{\sigma(2)}, \tau(1))=(1,2) \quad \rightarrow \quad(\overline{\sigma(3)}, \tau(2))=(1,1) \quad \rightarrow \\
& (\overline{\sigma(4)}, \tau(3))=(2,2) \quad \rightarrow \quad(\overline{\sigma(5)}, \overline{\tau(1)})=(1,1) \quad \rightarrow \quad(\sigma(1), \overline{\tau(2)})=(1,1) \quad \rightarrow \\
& (\sigma(2), \overline{\tau(3)})=(2,1) \quad \rightarrow \quad(\sigma(3), \tau(1))=(2,2) \quad \rightarrow \quad(\sigma(4), \tau(2))=(1,1) \quad \rightarrow \\
& (\sigma(5), \tau(3))=(2,2) \quad \rightarrow \quad(\overline{\sigma(1)}, \overline{\tau(1)})=(2,2),
\end{aligned}
$$

and from this point we will obtain the opposite orbit to the last $\ell=15$ iterates. In this case, the number of negative orientations is 5 .

Once we have described how to count the orientations in $L_{1}$, for the set $L_{2}$ that has subrectangles of type $\left(I^{\prime}\right)_{i(\bmod (q))}^{s} \times\left(J^{\prime}\right)_{i(\bmod (p))}^{t}$, with $s, t \in\{1,2\}$, we proceed in a similar way to that described for $L_{1}$. Since we have assumed that $f_{1}\left(I_{i}\right)=J_{i}^{\prime}$ and $f_{2}\left(J_{i}\right)=I_{i}^{\prime}$, we write $f_{2}\left(J_{1}^{1}\right) \times f_{1}\left(I_{1}^{1}\right)=\left(I^{\prime}\right)_{1}^{\mu(0)} \times\left(J^{\prime}\right)_{1}^{\rho(0)}$, for suitable $\mu(0), \rho(0) \in$ $\{1,2\}$. Now, we have to define $\mu(s), s=1, \ldots, q$ as the value in $\{1,2\}$ for which $\left(f_{2} \circ f_{1}\right)^{s}\left(\left(I^{\prime}\right)_{1}^{\mu(0)}\right)=\left(I^{\prime}\right)_{1+s(\bmod (q))^{\prime}}^{\mu(s)}$ and, similarly, $\rho(t) \in\{1,2\}$ will be characterized by $\left(f_{1} \circ f_{2}\right)^{t}\left(\left(J^{\prime}\right)_{1}^{\rho(0)}\right)=\left(J^{\prime}\right)_{1+t(\bmod (p))}^{\rho(t)}$, for $t=1, \ldots, p$. Notice that $\mu(q)=\overline{\mu(0)}$ and $\rho(p)=\overline{\rho(0)}$. By extending $\mu$ and $\rho$ to all positive integers, for counting the negative orientations we will have to pick the pairs $(\mu(i), \rho(i))$ with $\mu(i) \neq \rho(i)$, for $i=1, \ldots, \ell$.

2. Case $f_{1}(A) \cap \operatorname{Int}(B) \neq \varnothing$. This means that $p=q=\ell$, in fact $f_{1}(A)=B$ and $f_{2}(B)=A$. Firstly, let us show that the point $(a, b) \in I_{1} \times J_{1}$, given by $a=I_{1}^{1} \cap I_{1}^{2}, b=$ $J_{1}^{1} \cap J_{1}^{2}$, is $F$-periodic and its (minimal) period $m$ can be $\ell$ or $2 \ell$. Indeed, it is clear that $F^{2 \ell}(a, b)=\left(\left(f_{2} \circ f_{1}\right)^{\ell}(a),\left(f_{1} \circ f_{2}\right)^{\ell}(b)\right)=(a, b)$ because $a$ and $b$ are fixed points of $\left(f_{2} \circ f_{1}\right)^{\ell}$ and $\left(f_{1} \circ f_{2}\right)^{\ell}$, respectively, as a consequence of the no mixing property satisfied by $A$ and $B$, and the fact that $\ell=p=q$. On the other hand, if $n<\ell$ were the order of $(a, b)$, then $(a, b)=F^{2 n}(a, b)=\left(\left(f_{2} \circ f_{1}\right)^{n}(a),\left(f_{1} \circ f_{2}\right)^{n}(b)\right)$ would imply that $\ell \mid n$, a contradiction. Therefore, $m$ is either $\ell$ or $2 \ell$.

Consequently, we must distinguish two cases according to whether the order $m$ of the aforementioned $F$-periodic $(a, b)$ is $\ell$ or $2 \ell$ :

(i.O) If $m=\ell$, we affirm that the number $k$ of rectangles in $L=\omega_{F}(x, y)$ is $k=$ $\ell$, and in fact $\omega_{F}(x, y)$ is composed of $\ell$ rectangles of type $I_{i} \times J_{j}$. To prove it, recall that we are assuming that $(x, y) \in R_{1}=I_{1}^{1} \times J_{1}^{1} \subset I_{1} \times J_{1}$. Notice that $F^{\ell}(a, b)=(a, b) \in I_{1} \times J_{1}$, so $F^{\ell}\left(I_{1}^{1} \times J_{1}^{1}\right)=I_{1}^{i} \times J_{1}^{j}$ for some $i, j \in\{1,2\}$. If $i=j=1$, then we would have $F^{2 \ell}\left(I_{1}^{1} \times J_{1}^{1}\right)=F^{\ell}\left(I_{1}^{1} \times J_{1}^{1}\right)=I_{1}^{1} \times J_{1}^{1}$, but at the same time, for the decomposition of $A$ and $B$ obtained for their no mixing property, $F^{2 \ell}\left(I_{1}^{1} \times J_{1}^{1}\right)=\left(f_{2} \circ f_{1}\right)^{\ell}\left(I_{1}^{1}\right) \times\left(f_{1} \circ f_{2}\right)^{\ell}\left(J_{1}^{1}\right)=I_{1}^{2} \times J_{1}^{2}$, it is impossible. 
If $i=j=2$, now $F^{\ell}\left(I_{1}^{1} \times J_{1}^{1}\right)=I_{1}^{2} \times J_{1}^{2}$ and since also $F^{2 \ell}\left(I_{1}^{1} \times J_{1}^{1}\right)=I_{1}^{2} \times J_{1}^{2}$, we would obtain $F^{s \ell}\left(I_{1}^{2} \times J_{1}^{2}\right)=I_{1}^{2} \times J_{1}^{2}$ for all $s \geq 1$, a new contradiction (at least, $\left.F^{2 \ell}\left(I_{1}^{2} \times J_{1}^{2}\right)=I_{1}^{1} \times J_{1}^{1}\right)$. Therefore, $i \neq j$, which implies that either $F^{\ell}\left(I_{1}^{1} \times\right.$ $\left.J_{1}^{1}\right)=I_{1}^{1} \times J_{1}^{2}$ or $F^{\ell}\left(I_{1}^{1} \times J_{1}^{1}\right)=I_{1}^{2} \times J_{1}^{1}$. For instance, if $F^{\ell}\left(I_{1}^{1} \times J_{1}^{1}\right)=I_{1}^{1} \times J_{1}^{2}$, then $F^{3 \ell}\left(I_{1}^{1} \times J_{1}^{1}\right)=F^{2 \ell}\left(I_{1}^{1} \times J_{1}^{2}\right)=I_{1}^{2} \times J_{1}^{1}$, and thus all the rectangle $I_{1} \times J_{1}$ is included in $\operatorname{Orb}_{F}\left(I_{1}^{1} \times J_{1}^{1}\right)$. From here, and taking into account that an entire rectangle only contains at most a unique point of $\operatorname{Orb} F(a, b)$, we deduce that $|L|=\ell$, and $\omega_{F}(x, y)$ is composed of $k=\ell$ rectangles of type $I_{i} \times J_{j}$. Even more, its distribution follows the pattern of Distribution D1, and with equivalence we can reorder the intervals in such a way that $f_{1}\left(I_{i}\right)=J_{i+1(\bmod p)}$ and $f_{2}\left(J_{i}\right)=$ $I_{i+1(\bmod p)}$ for $i=1, \ldots, p$. See Figure 16.
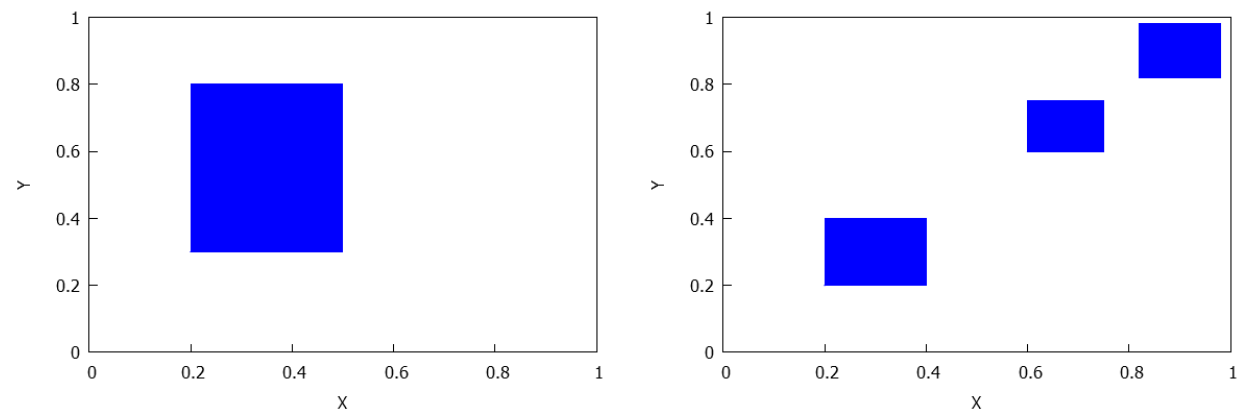

Figure 16. Case (i.O) corresponding to $\ell=1$ and $\ell=3$, whenever $f_{1}(A) \cap \operatorname{Int}(B) \neq \varnothing, A$ and $B$ no mixing.

Finally, notice that only the case $\ell$ odd is permitted here, since if $\ell$ is even, we would have $(a, b)=F^{\ell}(a, b)$ and then $\left(f_{2} \circ f_{1}\right)^{\frac{\ell}{2}}(a)=a,\left(f_{1} \circ f_{2}\right)^{\frac{\ell}{2}}(b)=$ $b$, impossible (realize that $a$ and $b$ are $\ell$-periodic points of $f_{2} \circ f_{1}$ and $f_{1} \circ f_{2}$, respectively).

(ii.E) If $m=2 \ell$, we assert that $|L|=4 \ell$ and, to be more precise, $L=\omega_{F}(x, y)$ is composed of $4 \ell$ rectangles having type $I_{i}^{u}, J_{j}^{v}$ for some $i, j \in\{1, \ldots, 2 \ell\}, u, v \in$ $\{1,2\}$, such that each of them are included in $I_{i} \times J_{j}$, with a common vertex given by a point of the orbit of $(a, b)$. To prove it, first notice that $\operatorname{Orb}_{F}(a, b)=$ : $\left\{\left(x_{i}, y_{i}\right): i=1, \ldots, 2 \ell\right\} \subseteq \operatorname{Orb}_{\left(f_{2} \circ f_{1}\right)}(a) \times \operatorname{Orb}_{\left(f_{1} \circ f_{2}\right)}(b)$, so from Lemma 5 and Theorem 3-(2) we deduce that for any $i \in\{1, \ldots, 2 \ell\}$ there exist $r, s \in\{1, \ldots, 2 \ell\}$, $r \neq s$, such that $x_{i}=x_{r}, y_{i}=y_{s}$. This implies that the $F$-orbit of $(a, b)$ is included in $2 \ell$ entire rectangles of type $I_{r} \times J_{s}$. On the other hand, $F^{2 \ell}\left(I_{1}^{1} \times J_{1}^{1}\right)=$ $\left(f_{2} \circ f_{1}\right)^{\ell}\left(I_{1}^{1}\right) \times\left(f_{1} \circ f_{2}\right)^{\ell}\left(J_{1}^{1}\right)=I_{1}^{2} \times J_{1}^{2}$, and $F^{j}\left(I_{1} \times J_{1}\right) \cap\left(I_{1} \times J_{1}\right)=\varnothing$ for $j=1, \ldots, \ell$ because $F^{j}(a, b) \neq(a, b)$ for $j \in\{1, \ldots, \ell\}$ and, according to the distribution provided for Theorem 3 to the orbit of $(a, b)$, each entire rectangle $I_{i} \times J_{j}$ only contains at most a unique point of $\operatorname{Orb}_{F}(a, b)$. All the above details allow us to conclude that $k=|L|=4 \ell$ and each rectangle $I_{i} \times J_{j}$ containing a point of $\operatorname{Orb}_{F}(a, b)$ is divided into two different subintervals with disjoint interiors and a point in $\operatorname{Orb}_{F}(a, b)$ as common vertex, see Figure 17, and therefore we obtain a structure of the type described in Figure 15.

Observe that if the position of the intervals is shifted, for example if $I_{i}^{1}>I_{i}^{2}$, for any $i$, then the orientation of every structure in the column $i$ changes. This case is the same as that obtained in Distribution D2, but changing the entire rectangle by two subrectangles of the type given in Figure 15. 

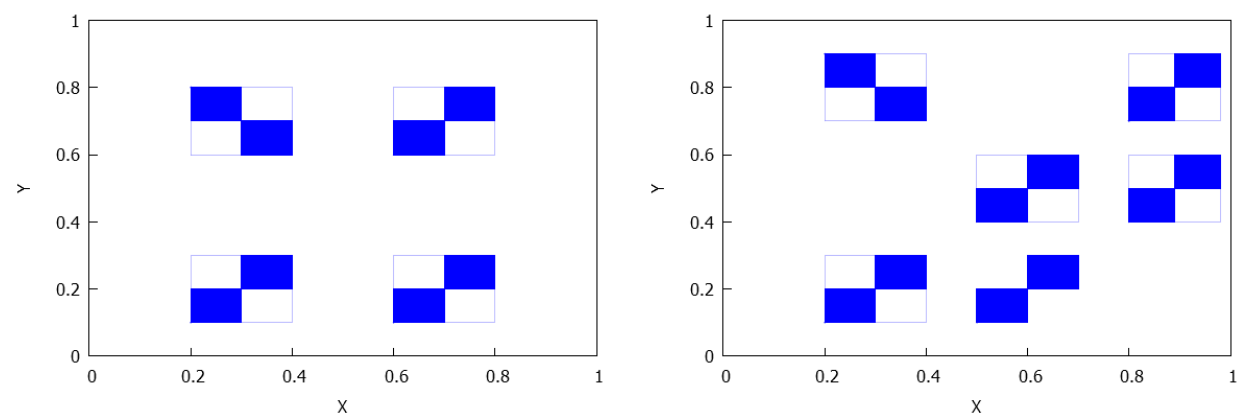

Figure 17. Case (ii.E) corresponding to $m=2 \ell=4$ and $m=2 \ell=6$, whenever $f_{1}(A) \cap \operatorname{Int}(B) \neq \varnothing$, $A$ and $B$ no mixing; here the number of rectangles is $k=4 \ell$ in each figure.

\subsection{The Converse Result for the Non-Empty Interior Case}

Until now, we have described the structure of $\omega$-limit sets of Cournot maps with nonempty interior. Of course, given one of these structures $L$, up to equivalences, it is natural to ask whether it is possible or not to find a Cournot map $F$ and a point $(x, y) \in I^{2}$ such that $\omega_{F}(x, y)=L$. The answer is the affirmative. The general strategy for this construction relies on the fact that we can take advantage of our knowledge of interval maps $f: I \rightarrow I$ exhibiting union of intervals $\bigcup_{i=1}^{m} I_{i}$ as $\omega$-limit sets for suitable points $x \in I$. For instance, for any interval $J=[a, b]$, there is a continuous map $f: J \rightarrow J$ with $f(a)=a$ and $f(b)=b$ such that $f$ is topologically mixing (see ([1], [p. 162])).

In order to illustrate it, we do the corresponding converse result for Distributions D1 (with $k$ odd), with the hope that the other cases can be managed for the reader without any difficulty.

(D1) Assume that $L=\bigcup_{i=1}^{k}\left(I_{i} \times J_{i}\right), k$ odd, and $I_{i}, J_{i}$ verify $(D 1)$, that is:

$$
\begin{aligned}
& L=\bigcup_{i=1}^{k} R_{i}=\bigcup_{i=1}^{k}\left(I_{\alpha_{i}} \times J_{\beta_{i}}\right), \text { with } I_{\alpha_{i}} \neq I_{\alpha_{j}}, J_{\beta_{i}} \neq J_{\beta_{j}}, \text { if } i \neq j, \\
& \pi_{1}(L)=\bigcup_{i=1}^{k} I_{\alpha_{i}}=A, \pi_{2}(L)=\bigcup_{i=1}^{k} J_{\beta_{i}}=B .
\end{aligned}
$$

To simplify the notation, let $\alpha_{i}=i$ and $\beta_{j}=j$, that is,

$$
\begin{aligned}
& A:=\bigcup_{i=1}^{k} \pi_{1}\left(R_{i}\right)=\bigcup_{i=1}^{k} I_{i}, \\
& B:=\bigcup_{i=1}^{k} \pi_{2}\left(R_{i}\right)=\bigcup_{i=1}^{k} J_{i} .
\end{aligned}
$$

Write $I_{i}=\left[a_{i(1)}, a_{i(2)}\right], J_{i}=\left[b_{i(1)}, b_{i(2)}\right]$. We then define $f_{1} \in C(I, I)$ in such a way that

$$
f_{1}\left(\left\{a_{i(1)}, a_{i(2)}\right\}\right)=b_{i+1(1)}, f_{i}\left(\frac{a_{i(1)}+a_{i(2)}}{2}\right)=b_{i+1(2)},
$$

and linear in the rest of $I_{i}, i=1, \ldots, k$. Also, we define a map $f_{2} \in C(I, I)$ holding $f_{2}\left(J_{i}\right)=$ $I_{i+1}$ in a linear way (change of variables) for $i=1, \ldots, k$. Then, it is straightforward to see that $\operatorname{Orb}_{F}\left(I_{1} \times J_{1}\right)=L$. Moreover, $\left(f_{2} \circ f_{1}\right)\left(I_{i}\right)=I_{i+2 \bmod (k)},\left(f_{1} \circ f_{2}\right)\left(J_{i}\right)=J_{i+2 \bmod (k)}$, $i \in\{1, \ldots, k\}$. Since $k$ is odd, from the definitions of $f_{j}$ we deduce that $f_{2} \circ f_{1}$ is mixing on $A, f_{1} \circ f_{2}$ is mixing on $B$, and $f_{1}(A)=B, f_{2}(B)=A$. According to Theorem 2 , there exists $(x, y) \in I^{2}$ such that $\omega_{F^{2 k}}(x, y)=I_{1}$. Then $\omega_{F}(x, y)=\operatorname{Orb}\left(I_{1} \times J_{1}\right)=L$.

\section{Applications to Economic Models}

In the seminal paper of Day [19] it was highlighted that complex behavior that can be described as chaotic can emerge from simple economic structures. This fact has had as a consequence for the increasing interest in economic dynamics, which is supported by the large amount of related literature published since then.

In this frame, oligopoly structure plays an important role. The assumption that only a few firms can compete makes sense in some markets in which the goods are perfectly substitutable [20]. In the decision process, each firm adjusts its output (product decision) 
based on the expectation over the other firm's output decisions. Under this premise, firms take their decisions simultaneously. In an oligopoly the supply side is (not perfectly) competitive-as we often say "Cournot competition". A duopoly is an oligopoly in which the number of firms is equal to two. Following Friedman [21], "a duopoly is a market in which two firms sell a product to a large number of consumers. Each consumer is too small to affect the market price for the product, that is, on the buyers' side, the market is competitive."

Different theoretical models of duopoly have been considered [21]. In 1838, Cournot [22] proposed a non-collusive duopoly model. In this duopoly model, firms decide their output levels simultaneously, that is, firms compete in quantities and it is assumed that price is given by the market. Moreover, each firm seeks to maximize the profit taking into account the decision of the competitor. Cournot duopoly was considered in [17], where the complexity of the dynamics was established. Later, Dana and Montrucchio [13] gave more details about the dynamical properties of the Cournot model. More recently, Pražák and Kovárník have considered in [15] a nonlinear inverse demand function and have shown bifurcation diagrams of the duopoly model.

Antitriangular maps are closely related to Cournot duopoly models. In this section we deal with the type of $\omega$-limits of several Cournot duopoly models in which dynamics is led by antitriangular maps.

Let us notice the relevance of this topic by highlighting its possible interest in implications for strategic decisions. In this direction, cyclical phenomena have an important role in economics in the analysis of the dynamics and fluctuations of the economic activity in a model. A cycle of length $n$ is the orbit of a periodic point. In the matter at hand, that is, $\omega$-limits of non-empty interior of Cournot maps, the full description of the structure is useful in the sense that it provides information about chaotic orbits (realize that $\omega$-limits with non-empty interior appear when the Cournot map $F$ is chaotic). A cyclical movement appears if, in the definition of cycle, we replace points by rectangles, since in each step the point of the chaotic orbit belongs to the corresponding rectangle and this turns on the fact that in each step we have boundaries (restrictions) for the values of the outputs because the boundaries of the rectangles limit the output values of the production firms when initial conditions have been fixed. In this way, the restriction of the regions of $\omega$-limits in Cournot duopolies plays an important role in the analysis of dynamics. Therefore, the distribution of the $\omega$-limits gives us information about the possible layouts in the rectangles giving the feasible region in which both firms take their output values, the rectangles being covered following a "cyclical process".

\subsection{Puu's Duopoly}

The following duopoly model was proposed by Puu [23]. In this model, the goods produced for both firms are equivalent and the demand function is isoelastic. It is assumed that the price is given by

$$
p=\frac{1}{x+y}
$$

where $x$ and $y$ are the outputs of firms $X$ and $Y$ respectively. The costs are assumed to be linear with the form $c_{1}(x)=c_{1} x$ and $c_{2}(y)=c_{2} y$, where $c_{1}$ and $c_{2}$, the marginal costs, will be the model parameters. Obviously, now profits are given by

$$
\begin{aligned}
& \Pi_{1}=\frac{x}{x+y}-c_{1} x, \\
& \Pi_{2}=\frac{y}{x+y}-c_{2} y .
\end{aligned}
$$

Considering that the objective of the firms is to maximize their own profits, the equations $\frac{\partial \Pi_{1}}{\partial x}=0$ and $\frac{\partial \Pi_{2}}{\partial y}=0$ give us

$$
x=\sqrt{\frac{y}{c_{1}}}-y,
$$




$$
y=\sqrt{\frac{x}{c_{2}}}-x
$$

Finally, the reaction map is

$$
F(x, y)=\left(f_{2}(y), f_{1}(x)\right)=\left(\max \left\{\sqrt{\frac{y}{c_{1}}}-y, 0\right\}, \max \left\{\sqrt{\frac{x}{c_{2}}}-x, 0\right\}\right),
$$

which is an antitriangular map in which has been introduced the max function due to the fact that productions cannot be negative. It is assumed without loss of generality that $c_{1} \geq c_{2}$, and we can compute $\omega$-limit sets, see some examples in Figure 18 (we have used the software Mathematica for generating Figures 18-23). More details about the dynamics of the model can be found in [24].
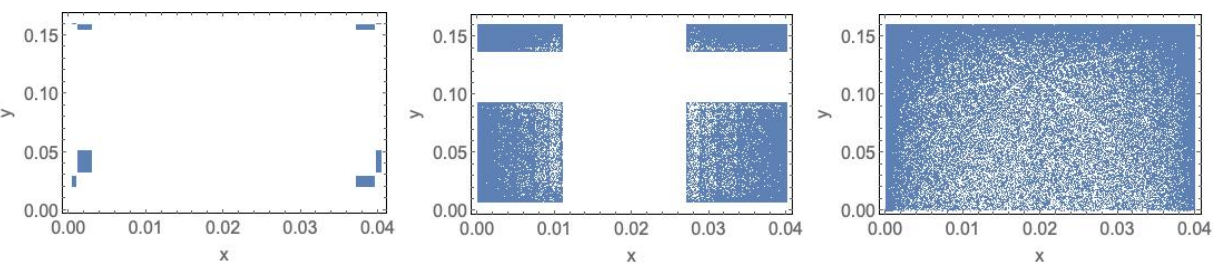

Figure 18. $\omega$-limit sets for Cournot Puu's model for $c_{2}=1$ and $c_{1}=6.194$ (left), $c_{1}=6.243$ (center) and $c_{1}=6.25$ (right) taking as initial conditions $\left(x_{0}, y_{0}\right)=(0.01,0.0011)$. We have computed orbits of length equal to 200,000 and we have drawn the last 100,000 points.

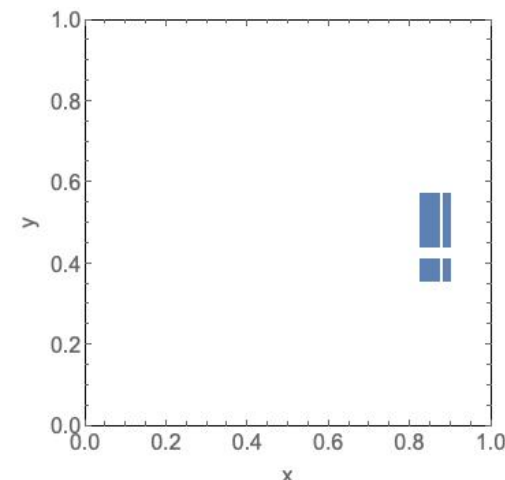

(a)

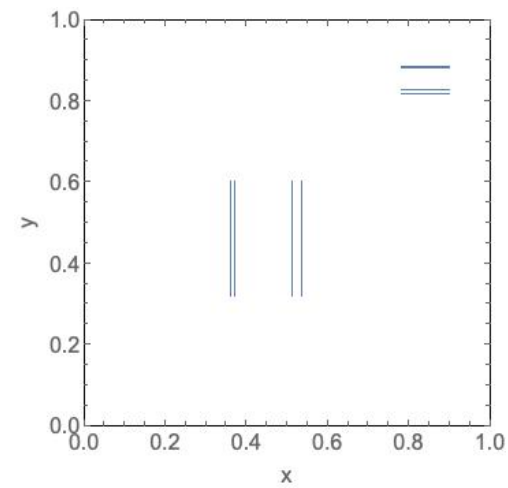

(d)

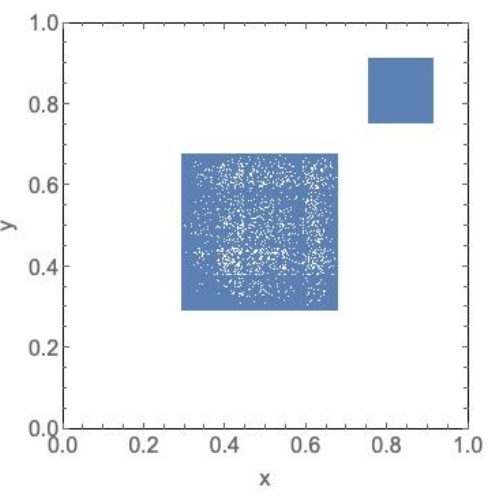

(b)

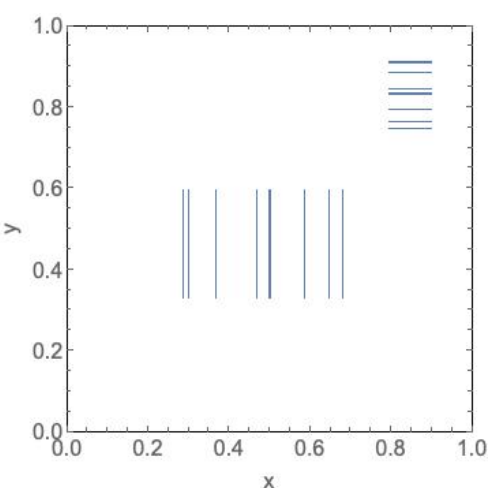

(e)

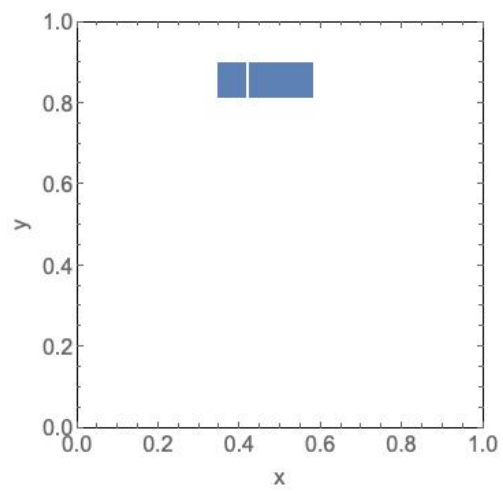

(c)

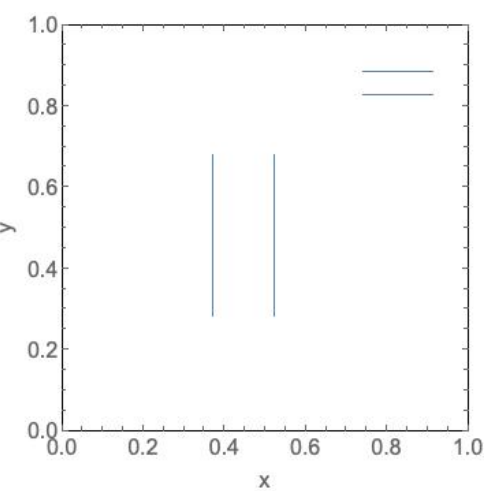

(f)

Figure 19. $\omega$-limit sets for Kopel's model for $\lambda_{1}=\lambda_{2}=1$. We have computed orbits or length equal to 200000 and we have plotted the last 100000. $\left(x_{0}, y_{0}\right)$ are the initial conditions. The parameter values for each figure follows. (a) $\mu_{1}=3.6$, $\mu_{2}=3.95, x_{0}=0.539815$ and $y_{0}=0.933646 ;(\mathbf{b}) \mu_{1}=3.65, \mu_{2}=3.65, x_{0}=0.539815, y_{0}=0.933646 ;(\mathbf{c}) \mu_{1}=3.85, \mu=3.6$, $x_{0}=0.539815, y_{0}=0.933646 ;(\mathbf{d}) \mu_{1}=3.6, \mu_{2}=3.55, x_{0}=0.539815, y_{0}=0.933646 ;(\mathbf{e}) \mu_{1}=3.6, \mu_{2}=3.65, x_{0}=0.539815$ and $y_{0}=0.933646$; (f) $\mu_{1}=3.65, \mu_{2}=3.55, x_{0}=0.539815, y_{0}=0.933646$. 


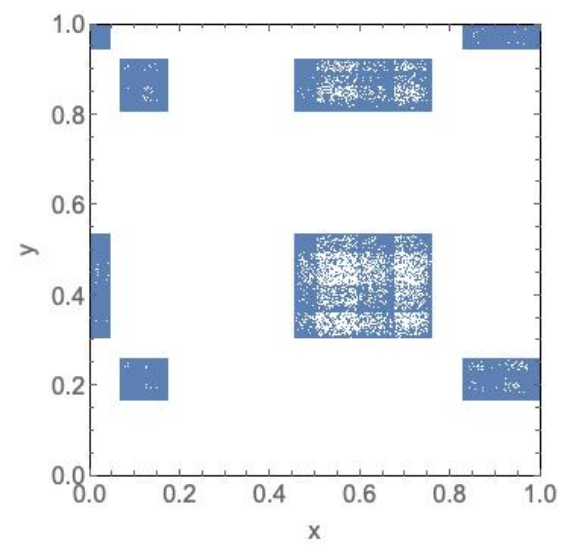

(a)

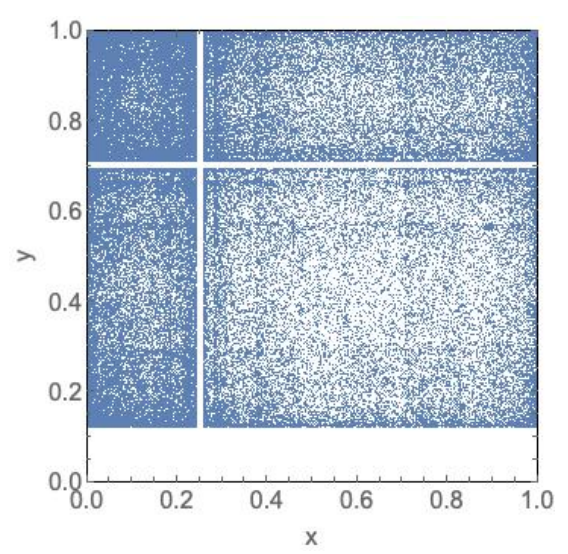

(d)

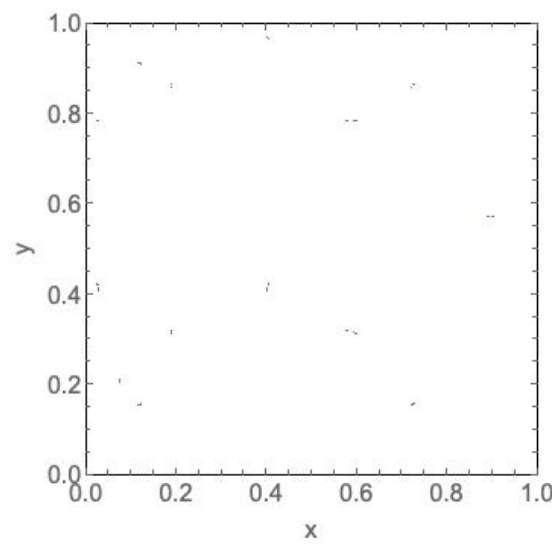

(b)

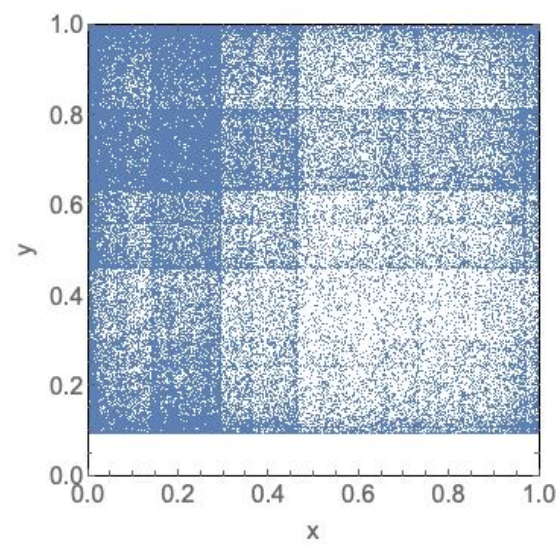

(e)

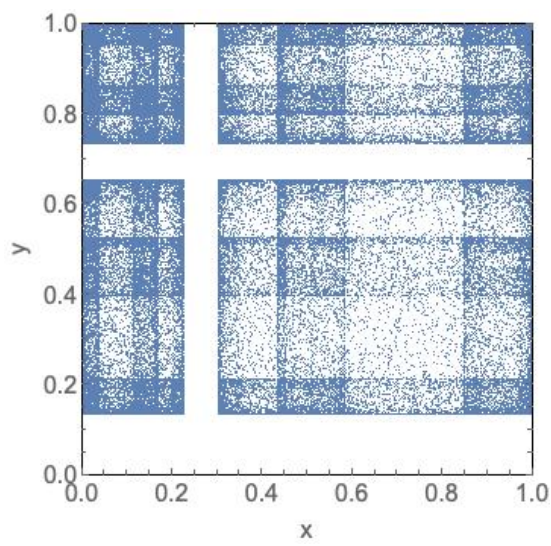

(c)

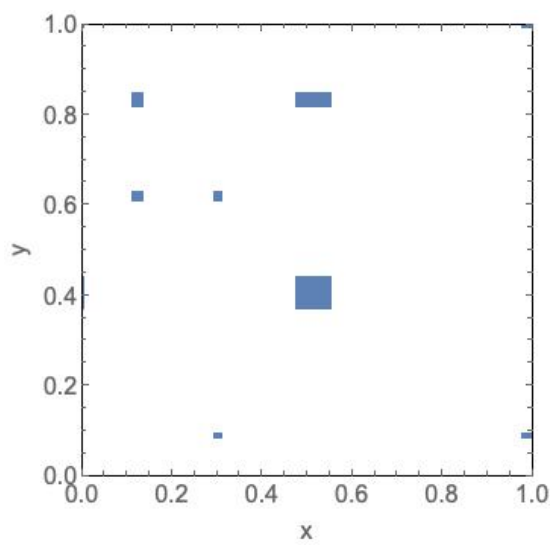

(f)

Figure 20. $\omega$-limit sets for Matsumoto-Nonaka's model. The value of the parameter $\alpha$ is $\alpha=1.7$. (a) $\beta=0.59, x_{0}=0.4$ and $y_{0}=1.2 ;(\mathbf{b})$ $\beta=0.605, x_{0}=0.4$ and $y_{0}=0.1 ;(\mathbf{c}) \mu=0.635, x_{0}=0.4$ and $y_{0}=0.2 ;(\mathbf{d}) \beta=0.65, x_{0}=0.4$ and $y_{0}=0.1 ;(\mathbf{e}) \beta=0.695, x_{0}=0.4$ and $y_{0}=0.2$; (f) $\beta=0.71, x_{0}=0.4$ and $y_{0}=0.7$. We have computed orbits of length 200,000 and we have drawn the last 100,000 points.

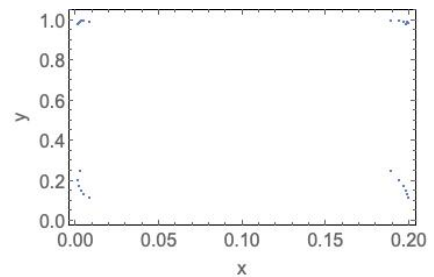

(a)

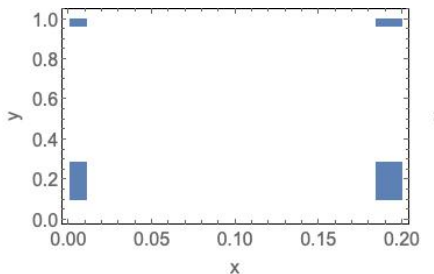

(b)

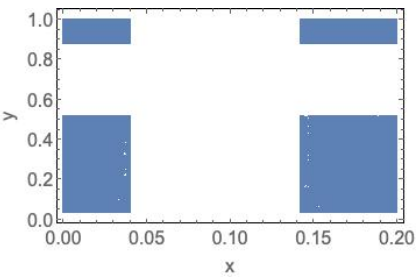

(c)

Figure 21. $\omega$-limit sets for Cournot duopoly when the competitors operate under capacity constraints. Initial conditions $x_{0}=0.2$ and $y_{0}=0.3$. The longitude orbit is equal to 200000 and the last 100000 points have been plotted. (a) $u=1$ and $v=8.137$; (b) $u=1$ and $v=8.151$; (c) $u=1$ and $v=8.193$. 

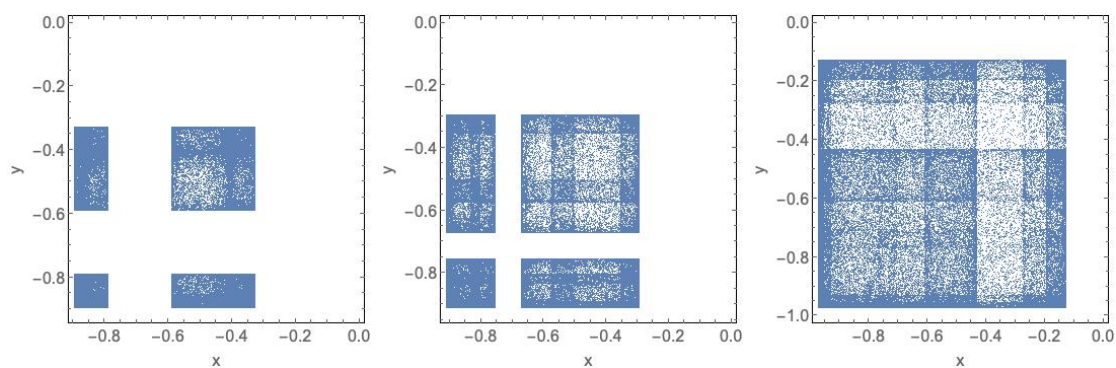

Figure 22. $\omega$-limit sets for $\mu=1.56, \mu=1.58$ and $\mu=1.66$ (left, center and right respectively) and initial conditions $x_{0}=-0.5$ and $y_{0}=-0.3$.
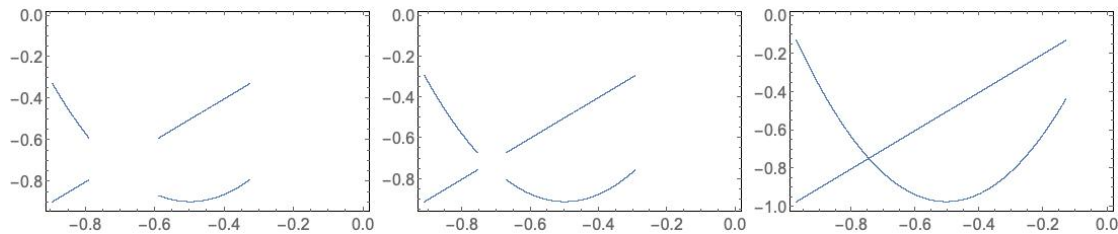

Figure 23. $\omega$-limit sets with empty interior for $\mu=1.56, x_{0}=-0.5$ and $y_{0}=-0.895647$ (left); $\mu=1.58, x_{0}=-0.5$ and $y_{0}=-0.911129$ (center); $\mu=1.66, x_{0}=-0.5$ and $y_{0}=-0.973056$ (right).

\subsection{Kopel's Duopoly}

M. Kopel introduced in [14] a general Cournot duopoly model in which two firms, $X$ and $Y$, plan their productions, $x_{t}, y_{t}$, at time $t$, following the system of difference equations:

$$
\begin{aligned}
& x_{t+1}=\max \left\{0,\left(1-\lambda_{1}\right) x_{t}+\lambda_{1} \mu_{1} y_{t}\left(1-y_{t}\right)\right\}, \\
& y_{t+1}=\max \left\{0,\left(1-\lambda_{2}\right) y_{t}+\lambda_{2} \mu_{2} x_{t}\left(1-x_{t}\right)\right\},
\end{aligned}
$$

where $\lambda_{i} \in[0,1]$ and $\mu_{i}>0$ for $i=1,2$. The positive parameters $\mu_{i}$ measure the intensity of the effect that one firm's action has on the other firm, see also [25] for more details. Then, the two-dimensional map

$$
R_{\lambda_{1}, \lambda_{2}, \mu_{1}, \mu_{2}}(x, y):=\left(\max \left\{0,\left(1-\lambda_{1}\right) x+\lambda_{1} \mu_{1} y(1-y)\right\}, \max \left\{0,\left(1-\lambda_{2}\right) y+\lambda_{2} \mu_{2} x(1-x)\right\}\right),
$$

describes the simultaneous choices of both firms.

Observe that taking $\lambda_{1}=\lambda_{2}=1$, a Cournot duopoly model is obtained, given as

$$
F(x, y)=\left(\max \left\{0, \mu_{1} y(1-y)\right\}, \max \left\{0, \mu_{2} x(1-x)\right\}\right) \text {. }
$$

In Figure 19, we have shown some $\omega$-limits for the map (7). We have selected some of them. To get it, we have used random initial conditions, with 6 decimal digits by default.

\subsection{Matsumoto-Nonaka's Model}

In [26] a two-market model consisting of two firms that produce differentiated goods is introduced, see also [27]. The first firm produces good $x$ in the first market and the second one produces good $y$ in the second market. In this model, different externalities affect the dynamics.

As in the previous subsection, in [26] a more general model is introduced. Nevertheless, we are interested in the particular case in which inverse demand functions are given by

$$
\begin{aligned}
& p_{1}(x, y)=(\alpha-1)^{2}-\frac{x}{2}+(\alpha y)^{2}, \\
& p_{2}(x, y)=1-\frac{y}{2}+(\beta x)^{2},
\end{aligned}
$$


where $p_{1}$ and $p_{2}$ are the market prices of goods $x$ and $y$, respectively, and $\alpha \in[1,2] \subset \mathbb{R}$ and $\beta \in[0,2] \subset \mathbb{R}$. Each firm decides future production depending on the other firm's choice and production externalities. Cost functions are given by

$$
\begin{aligned}
& C_{1}(x, y)=2(\alpha-1) \alpha x y, \\
& C_{2}(x, y)=2 \beta x y,
\end{aligned}
$$

and the profit function of each firm is given by

$$
\begin{aligned}
& \Pi_{1}(x, y)=p_{1}(x, y) x-C_{1}(x, y)=\left((\alpha-1)^{2}-\frac{x}{2}+\alpha^{2} y^{2}\right) x-2(\alpha-1) \alpha x y, \\
& \Pi_{2}(x, y)=p_{2}(x, y) y-C_{2}(x, y)=\left(1-\frac{y}{2}+\beta^{2} x^{2}\right) y-2 \beta x y .
\end{aligned}
$$

As assumed above, each firm tends to maximize its profit. In order to do this the firms can choose their production levels, which would affect to the other firm. The first firm maximizes the profit with respect to $x$ and the same occurs for the second firm with respect to $y$. The reaction functions of the firms are given by

$$
\begin{aligned}
& f_{\alpha}(y)=(\alpha y-\alpha+1)^{2}, \\
& g_{\beta}(x)=(\beta x-1)^{2} .
\end{aligned}
$$

Now, the function

$$
R_{\alpha, \beta}(x, y)=\left(f_{\alpha}(y), g_{\beta}(x)\right)=\left((\alpha y-\alpha+1)^{2},(\beta x-1)^{2}\right),
$$

is the reaction function for the outcome $(x, y)$. The iterations are given by

$$
\begin{aligned}
& x_{t+1}=f_{\alpha}\left(y_{t}\right)=\left(\alpha y_{t}-\alpha+1\right)^{2}, \\
& y_{t+1}=g_{\beta}\left(x_{t}\right)=\left(\beta x_{t}-1\right)^{2},
\end{aligned}
$$

that is,

$$
\left(x_{t+1}, y_{t+1}\right)=F_{\alpha, \beta}\left(x_{t}, y_{t}\right)=\left(f_{\alpha}\left(y_{t}\right), g_{\beta}\left(x_{t}\right)\right),
$$

where $\alpha \in[1,2] \subset \mathbb{R}$ and $\beta \in[0,2] \subset \mathbb{R}$. Thus, the reaction map $F_{\alpha, \beta}$ is a Cournot map from $[0,1] \times[0,1]$ into $[0,1] \times[0,1]$. In this situation, we obtain some $\omega$-limits, the results are shown in Figure 20.

\subsection{Cournot Duopoly When the Competitors Operate under Capacity Constraints}

In [28] A. Norin and T. Puu introduced a Cournot duopoly where the competitors have capacity constraints. The production cost of the firms are given by

$$
\begin{aligned}
& T C_{1}(x)=-\log \left(1-\frac{x}{u}\right), \\
& T C_{2}(y)=-\log \left(1-\frac{y}{v}\right),
\end{aligned}
$$

where $u$ and $v$ denote the capacity limits and $x$ and $y$ denote the supplies of the two competitors. Observe that when $x=y=0$ then the total costs are zero and tend to infinity when production approaches capacity limits. The price $p$ is given by $p=\frac{1}{x+y}$ and the profits are

$$
\begin{aligned}
& \Pi_{X}(x, y)=p \cdot x-T C_{1}(x)=\frac{x}{x+y}+\log \left(1-\frac{x}{u}\right), \\
& \Pi_{Y}(x, y)=p \cdot y-T C_{2}(y)=\frac{y}{x+y}+\log \left(1-\frac{y}{u}\right) .
\end{aligned}
$$


To maximize the profits (10) we get the following equations

$$
\begin{gathered}
\frac{\partial \prod_{X}}{\partial x}(x, y)=\frac{y}{(x+y)^{2}}-\frac{1}{u-x}=0, \\
\frac{\partial \prod_{Y}}{\partial y}(x, y)=\frac{x}{(x+y)^{2}}-\frac{1}{v-y}=0 .
\end{gathered}
$$

From the previous system of Equation (11), solving the first for $x$ and the second for $y$ (see [28] for details), the following reaction functions are obtained

$$
\begin{aligned}
& f_{u}(y)=\max \left\{0, \frac{1}{2} \sqrt{4 u y+5 y^{2}}-\frac{3}{2} y\right\} \\
& f_{v}(x)=\max \left\{0, \frac{1}{2} \sqrt{4 v x+5 x^{2}}-\frac{3}{2} x\right\},
\end{aligned}
$$

since clearly productions cannot be negative. Hence, the reaction map we construct is a Cournot map

$$
F_{u, v}(x, y)=\left(f_{u}(y), f_{v}(x)\right) .
$$

Different $\omega$-limits for the model are shown in Figure 21, where the initial conditions are $x_{0}=0.2$ and $y_{0}=0.3$. We have computed orbits of length equal to 200,000 and we have drawn the last 100,000 points.

\subsection{A Recurrent Class of Two-Dimensional Endomorphisms}

The class of maps given by $F(x, y)=(y, h(x))$, where $h(x)$ is a continuous function, piecewise continuously differentiable, has been considered among others in [29], and in this framework this kind of map provides us another different example to which we can apply this analysis.

Observe that $F^{2}(x, y)=(h(x), h(y))$. Following [29] we consider the map $h(x)=$ $-\alpha x+\beta g(\gamma x)$, where

$$
g(x)= \begin{cases}4 \mu x(1-x) & \text { if } 0<x<1 \quad(\mu>0), \\ 0 & \text { otherwise. }\end{cases}
$$

The parameters satisfy $0<\alpha<1,-1<\beta<0$ and $\gamma<0$. In our particular case, the parameters will use in the simulations are fixed, namely $\alpha=0.62386015, \beta=-0.790837347$ and $\gamma=-0.85446789$. See results in Figures 22 and 23.

\section{Some Advances in the Empty-Interior Case}

For a Cournot map, we already know the topological structure of an $\omega$-limit set with empty interior, see Theorem 1. Essentially, the situation is similar to the interval case, the $\omega$-limit set is a nowhere dense set. In the interval case this means that the set is totally disconnected. This changes for Cournot maps, we can find $\omega$-limit sets with empty interior and locally connected, even connected.

Our first example shows an $\omega$-limit set of empty interior homeomorphic to the circle $\mathbb{S}^{1}=\{z \in \mathbb{C}:|z|=1\}$. Consider a transitive map $f_{1}:[0,1] \rightarrow[0,1]$ with $f_{1}(0)=1$ and $f_{1}(1)=0$; for instance, take

$$
f_{1}(x)=\left\{\begin{array}{c}
1-2 x, \text { if } x \in\left[0, \frac{1}{2}\right] \\
T\left(2\left(x-\frac{1}{2}\right)\right), \text { if } x \in\left[\frac{1}{2}, 1\right]
\end{array}\right.
$$

where $T$ is the well-known tent map $T(x)=1-|1-2 x|, x \in[0,1]$.

$f_{1}$ being (totally) transitive, let $x_{0} \in[0,1]$ be a transitive point, that is, a point with orbit dense. Consider the map $F_{1}(x, y)=\left(y, f_{1}(x)\right)$. In this case, it is easily seen that

$$
\omega_{F_{1}}\left(\left(0, x_{0}\right)\right)=(\{0\} \times I) \bigcup(I \times\{1\}) \bigcup(\{1\} \times I) \bigcup(I \times\{0\}) .
$$


Therefore, $\omega_{F_{1}}\left(\left(0, x_{0}\right)\right)$ is homeomorphic to the unit circle. See Figure 24.
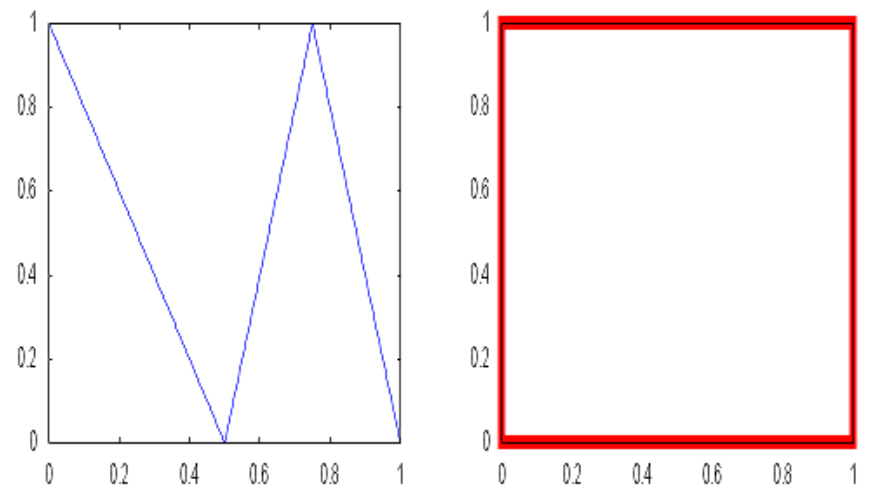

Figure 24. The map $f_{1}$ and the $\omega$-limit set $\omega_{F_{1}}\left(\left(0, x_{0}\right)\right)$.

As a second example, consider the transitive map $f_{2}:[0,1] \rightarrow[0,1]$ given by

$$
f_{2}(x)=\left\{\begin{array}{c}
2\left(x+\frac{1}{4}\right), \text { if } x \in\left[0, \frac{1}{4}\right], \\
1-2\left(x-\frac{1}{4}\right) \text { if } x \in\left[\frac{1}{4}, \frac{3}{4}\right], \\
2\left(x-\frac{3}{4}\right) \text { if } x \in\left[\frac{3}{4}, 1\right] .
\end{array}\right.
$$

Notice that $f_{2}$ is transitive but $f_{2}^{2}$ is not. Consider a point $y_{0}$ for which $\omega_{f_{2}}\left(y_{0}\right)=I$, and define the antitriangular map $F_{2}(x, y)=\left(y, f_{2}(x)\right)$. Now, a direct computation of the orbit of $\left(\frac{1}{2}, y_{0}\right)$ gives the limit set (see Figure 25)

$$
\omega_{F_{2}}\left(\frac{1}{2}, y_{0}\right)=\left(\left\{\frac{1}{2}\right\} \times I\right) \cup\left(I \times\left\{\frac{1}{2}\right\}\right) .
$$
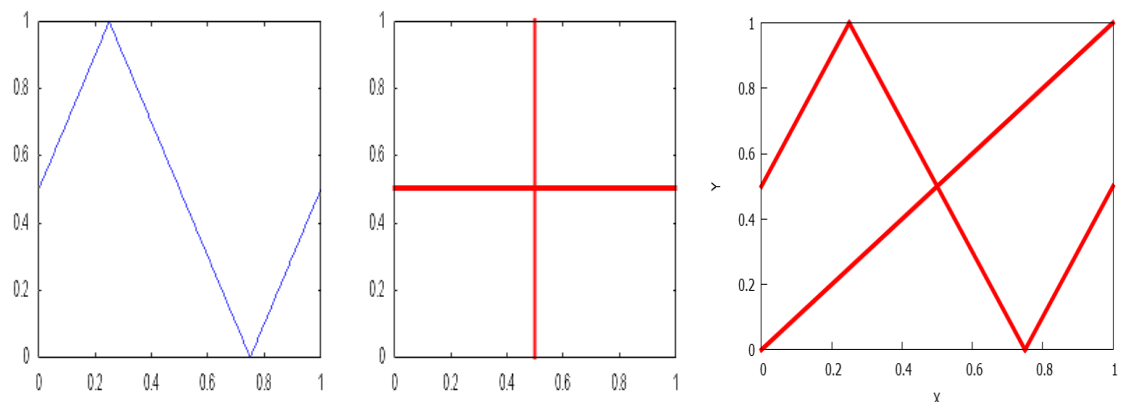

Figure 25. The map $f_{2}$ and the $\omega$-limit sets $\omega_{F_{2}}\left(\left(\frac{1}{2}, y_{0}\right)\right), \omega_{F_{2}}\left(\left(y_{0}, y_{0}\right)\right)$.

In view of the last example, we stress that it is possible to find $\omega$-limit sets of antitriangular maps consisting of the union of the diagonal and the graph of an interval map (for instance, see Figure 26).

Proposition 2. Let $f \in C(I, I)$ be a transitive map. Let $x_{0} \in I$ such that $\omega_{f}\left(x_{0}\right)=I$. Then,

$$
\Delta \cup \Gamma_{f}=\{(x, x): x \in I\} \cup\{(x, f(x)): x \in I\}
$$

can be realized as the $\omega$-limit set of the Cournot map $F(x, y)=(y, f(x))$.

Proof. It suffices to consider the point $\left(x_{0}, x_{0}\right)$ to obtain $\omega_{F}\left(x_{0}, x_{0}\right)=\Delta \cup \Gamma_{f}$. 


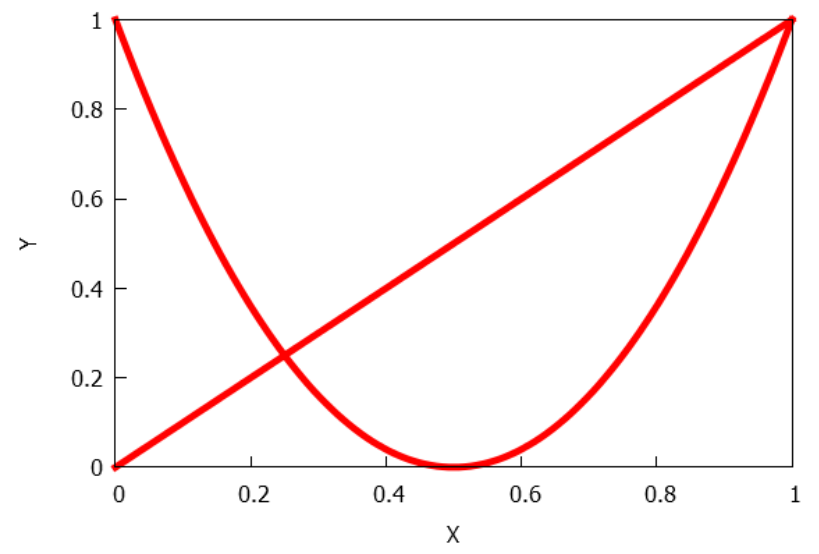

Figure 26. The transitive map $f(x)=4\left(x-\frac{1}{2}\right)^{2}$ originates the $\omega$-limit set $\omega_{F}\left(\left(x_{0}, x_{0}\right)\right)=\Delta \cup \Gamma_{f}$.

Remark 3. If in the above result, we choose a map $f \in C(I, I)$ and a point $x_{0}$ such that $\omega_{f}\left(x_{0}\right)$ is a Cantor set $C$; notice that we are able to find totally disconnected $\omega$-limit sets of the form $\{(x, x): x \in C\} \cup\{(x, f(x)): x \in C\}$. For instance, if we consider the well-known parabola $f(x)=\lambda_{\infty} x(1-x)$ of type $2^{\infty}$ (its set of periods is given by all the powers of 2 ), where $\lambda_{\infty}=$ $3.569 . .$. is the limit value of the period doubling bifurcation or route to chaos of the unimodal family of parabolas $f_{\lambda}(x)=\lambda x(1-x), \lambda \in[0,4]$ (for instance, see [30] for more information), we can choose a point $x_{0}$ in such a way that $\omega_{f}\left(x_{0}\right)=C_{0}$ is homeomorphic to the classical ternary Cantor set and, in this case for $F(x, y)=(y, f(x))$, we find that $\omega_{F}\left(x_{0}, x_{0}\right)=\{(x, x): x \in$ $\left.C_{0}\right\} \cup\left\{(x, f(x)): x \in C_{0}\right\}$, see Figure 27 .

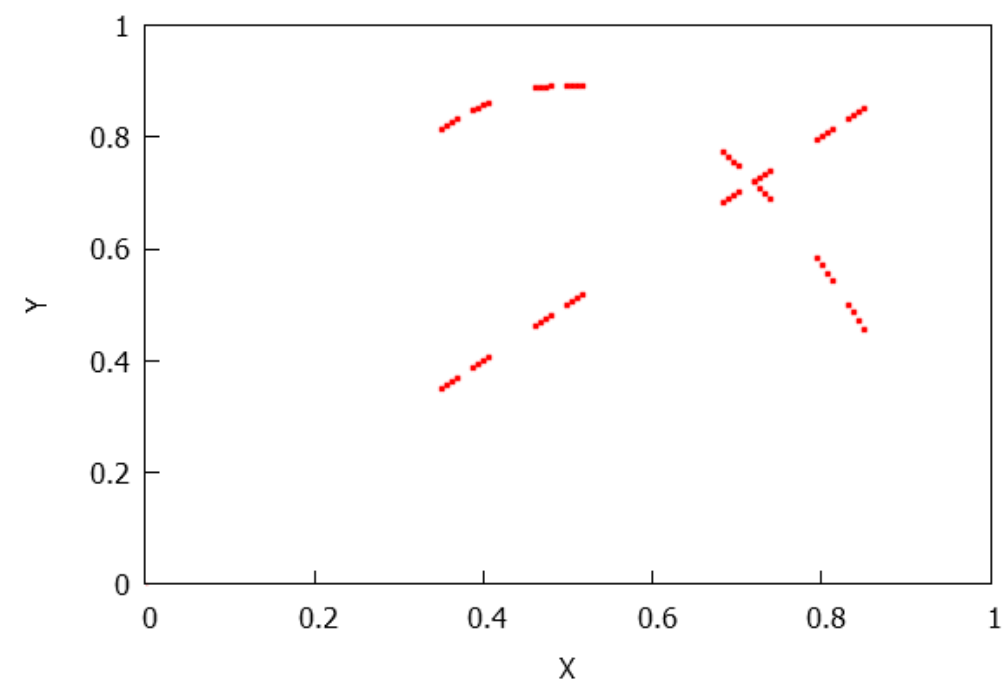

Figure 27. The transitive map $f(x)=\lambda_{\infty} x(1-x)$ originates the $\omega$-limit set $\omega_{F}\left(\left(x_{0}, x_{0}\right)\right)=\left.\left.\Delta\right|_{C} \cup \Gamma_{f}\right|_{C}$.

In the same direction, we find the following result on a peculiar type of $\omega$-limit set for Cournot maps (see Figure 28).

Proposition 3. Let $f \in C(I, I)$ be a totally transitive map. Let $\Gamma_{f}$ be its graph and $\Gamma_{f}^{*}=$ $\{(f(x), x): x \in I\}$. Then there exists an antitriangular map $F$ and a point $(x, y) \in I^{2}$ such that $\omega_{F}(x, y)=\Gamma_{f} \cup \Gamma_{f}^{*}$

Proof. Since $f$ is transitive, there is $x_{0} \in I$ such that $\omega_{f}\left(x_{0}\right)=I$. Consider the antitriangular map $F(x, y)=(f(y), f(x))$. Since $f$ is totally transitive, it is easy to check that $\omega_{f}\left(x_{0}, f\left(x_{0}\right)\right)=\Gamma_{f} \cup \Gamma_{f}^{*}$. 


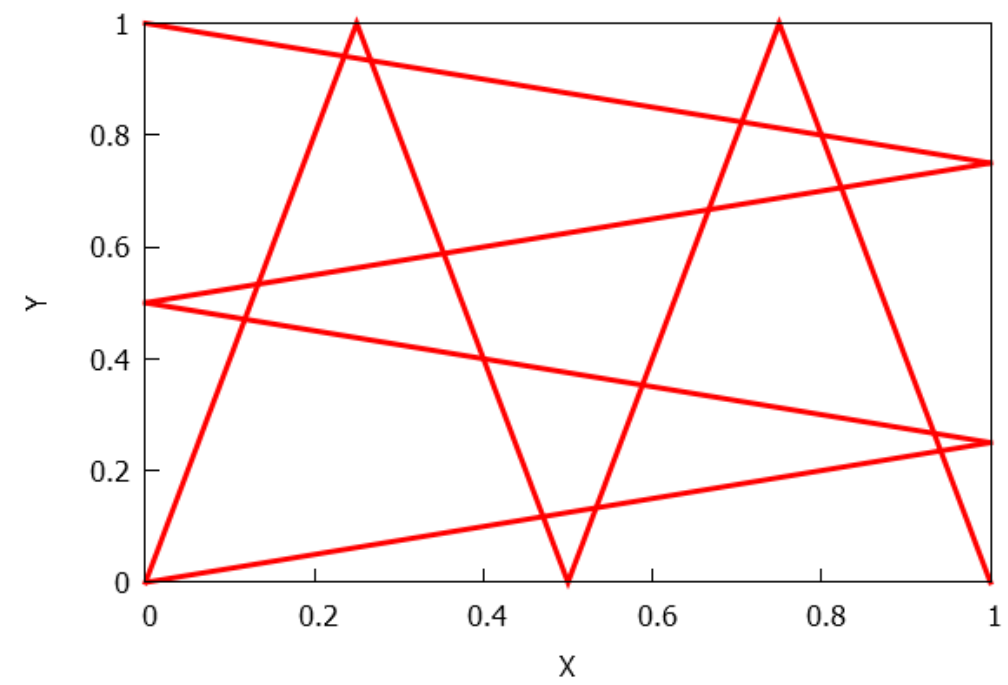

Figure 28. The graphs $\Gamma_{f}$ and $\Gamma_{f}^{*}$ corresponding to $f=T^{2}$, the second iterate of the tent map.

In our third example we stress the fact that, contrary to the first examples, there exist $\omega$-limit sets of antitriangular maps which are not connected. To see it, define the map (see Figure 29)

$$
f_{3}(x)=\left\{\begin{array}{c}
1-\frac{3}{2} x, \text { if } x \in\left[0, \frac{1}{3}\right], \\
f_{2}\left(3\left(x-\frac{1}{3}\right)\right), \text { if } x \in\left[\frac{1}{3}, \frac{2}{3}\right], \\
1-\frac{3}{2} x, \text { if } x \in\left[\frac{1}{3}, \frac{2}{3}\right]
\end{array}\right.
$$

where $f_{2}$ was defined in the second example. In this case, there exists $z_{0} \in\left[\frac{1}{3}, \frac{2}{3}\right]$ such that $\omega_{f_{3}}\left(z_{0}\right)=\left[\frac{1}{3}, \frac{2}{3}\right]$. By defining $F_{3}(x, y)=\left(y, f_{3}(x)\right)$, a direct inspection of the orbit of $\left(0, z_{0}\right)$ gives us the limit set

$$
\omega_{F_{3}}\left(\left(0, x_{0}\right)\right)=\left(\{0\} \times\left[\frac{1}{3}, \frac{2}{3}\right]\right) \bigcup\left(\left[\frac{1}{3}, \frac{2}{3}\right] \times\{1\}\right) \bigcup\left(\{1\} \times\left[\frac{1}{3}, \frac{2}{3}\right]\right) \bigcup\left(\left[\frac{1}{3}, \frac{2}{3}\right] \times\{0\}\right) .
$$
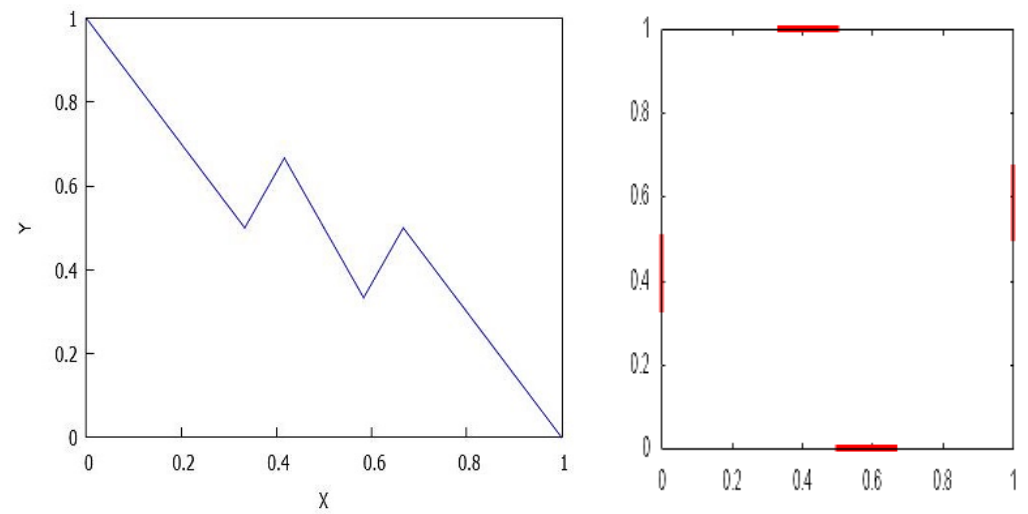

Figure 29. The map $f_{3}$ and, in red, the $\omega$-limit set $\omega_{F_{3}}\left(\left(0, z_{0}\right)\right)$.

Our following example exhibits an $\omega$-limit set with empty interior and homeomorphic to $C_{2}:=\left\{(x, y): x^{2}+y^{2}=1\right\} \cup\left\{(x, y):(x-2)^{2}+y^{2}=1\right\}$, namely $C_{2}$ is the union of two circles joined by a point. We define $F_{4}(x, y)=\left(y, f_{4}(x)\right)$, where

$$
f_{4}(x)=\left\{\begin{array}{c}
3 x, \text { if } x \in\left[0, \frac{1}{3}\right], \\
-3 x+2, \text { if } x \in\left[\frac{1}{3}, \frac{2}{3}\right], \\
3 x-2, \text { if } x \in\left[\frac{2}{3}, 1\right] .
\end{array}\right.
$$


Since $f_{4}$ is a transitive map, take a point $w_{0} \in I$ such that $\omega_{f_{4}}\left(w_{0}\right)=I$. Then by direct computation we find that

$$
\omega_{F_{4}}\left(\left(w_{0}, w_{0}\right)\right)=\Delta \cup \Gamma_{f_{4}}
$$

where $\Delta$ is the diagonal of the unit square and $\Gamma_{f_{4}}=\left\{\left(x, f_{4}(x)\right): x \in I\right\}$ is the graph of $f_{4}$ (see Figure 30).
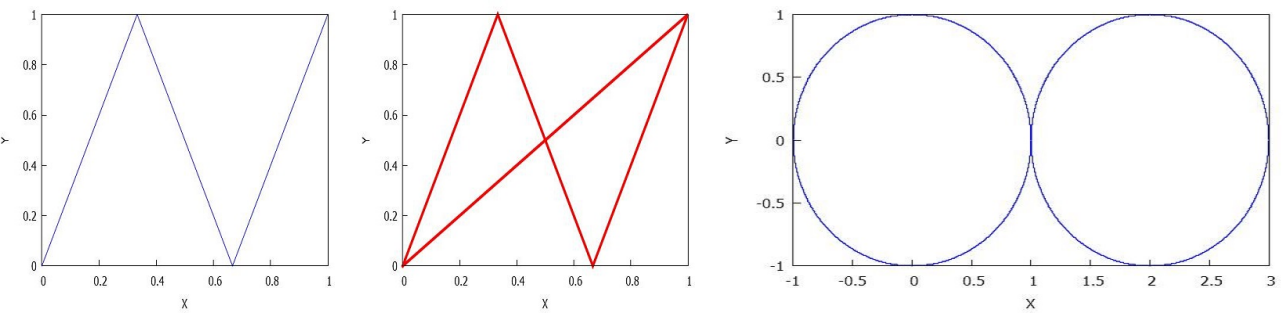

Figure 30. The map $f_{4}$ and the $\omega$-limit set $\omega_{F_{4}}\left(\left(w_{0}, w_{0}\right)\right)$ homeomorphic to $C_{2}$.

In fact, we can generalize the above example to find $\omega$-limit sets homeomorphic to the union of $n$-circles, $n \geq 2$, attach one of them to the other by a point, or

$$
C_{n}=\left\{(x, y) \in \mathbb{R}^{2}:(x-2 j)^{2}+y^{2}=1, \text { for some } j=0,1, \ldots, n-1\right\} .
$$

This is a substantial difference of the $\omega$-limit sets of antitriangular maps with respect to the one dimensional case - the limit sets can be connected and, at the same time, exhibit empty interior.

Proposition 4. For each $n \geq 1$, there exists an antitriangular map $\Phi_{n}$ such that $\omega_{\Phi_{n}}\left(X_{n}\right)$ is homeomorphic to $C_{n}$ for some $X_{n} \in I^{2}$.

Proof. We distinguish two cases according to the parity of $n$.

If $n=2 k$, define (see Figure 31 for $n=6$ )

$$
\varphi_{n}(x)=\left\{\begin{array}{c}
(n+1)\left(x-\frac{2 j}{n+1}\right), \text { if } x \in\left[\frac{2 j}{n+1}, \frac{2 j+1}{n+1}\right] \text { for } j=0,1, \ldots, \frac{n}{2} \\
1-(n+1)\left(x-\frac{2 j-1}{n+1}\right), \text { if } x \in\left[\frac{2 j-1}{n+1}, \frac{2 j}{n+1}\right] \text { for } j=1, \ldots, \frac{n}{2} .
\end{array}\right.
$$

Since $\varphi_{n}$ is transitive, take a point $x_{0} \in I$ for which $\omega_{\varphi_{n}}\left(x_{0}\right)=I$. Define $\Phi_{n}(x, y)=$ $\left(y, \varphi_{n}(x)\right)$. Then, as an immediate consequence of iterating $\left(x_{0}, x_{0}\right)$ successively and to consider the transitivity of the point $x_{0}$, we obtain

$$
\omega_{\Phi_{n}}\left(\left(x_{0}, x_{0}\right)\right)=\Delta \cup \Gamma_{\varphi_{n}}
$$

where $\Delta$ is the diagonal of the unit square and $\Gamma_{\varphi_{n}}=\left\{\left(x, \varphi_{n}(x)\right): x \in I\right\}$ is the graph of $\varphi_{n}$.
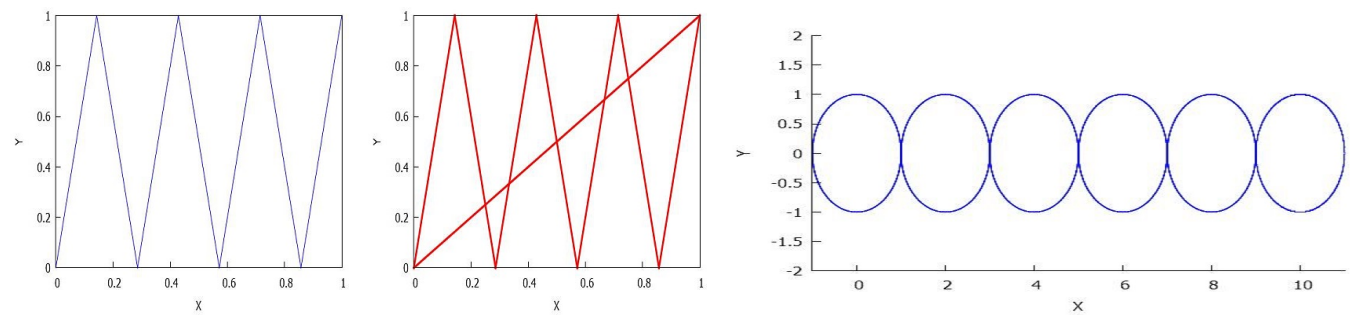

Figure 31. The map $\varphi_{6}$ and the $\omega$-limit set $\omega_{\Phi_{6}}\left(\left(x_{0}, x_{0}\right)\right)$ homeomorphic to $C_{6}$. 
For the odd case, first we define (see Figure 32)

$$
\varphi_{3}(x)=\left\{\begin{array}{c}
4 x, \text { if } x \in\left[0, \frac{1}{4}\right] \\
\frac{3}{2}-2 x, \text { if } x \in\left[\frac{1}{4}, \frac{1}{2}\right] \\
4 x-\frac{3}{2}, \text { if } x \in\left[\frac{1}{2}, \frac{5}{8}\right] \\
6-8 x, \text { if } x \in\left[\frac{5}{8}, \frac{3}{4}\right] \\
4 x-3, \text { if } x \in\left[\frac{3}{4}, 1\right]
\end{array}\right.
$$

With this function, we consider the antitriangular map $\Phi_{3}(x, y)=\left(y, \varphi_{3}(x)\right)$. Notice that $\varphi_{3}(x)$ is a transitive map, therefore there exists $w_{0} \in I$ such that $\omega_{\varphi_{3}}\left(w_{0}\right)=I$. With respect to $\Phi_{3}$, it is easily seen that $\omega_{\Phi_{3}}\left(w_{0}, w_{0}\right)=\Delta \cup \Gamma_{\varphi_{3}}$, where, again, $\Gamma_{\varphi_{3}}$ means the graph of $\varphi_{3}$.
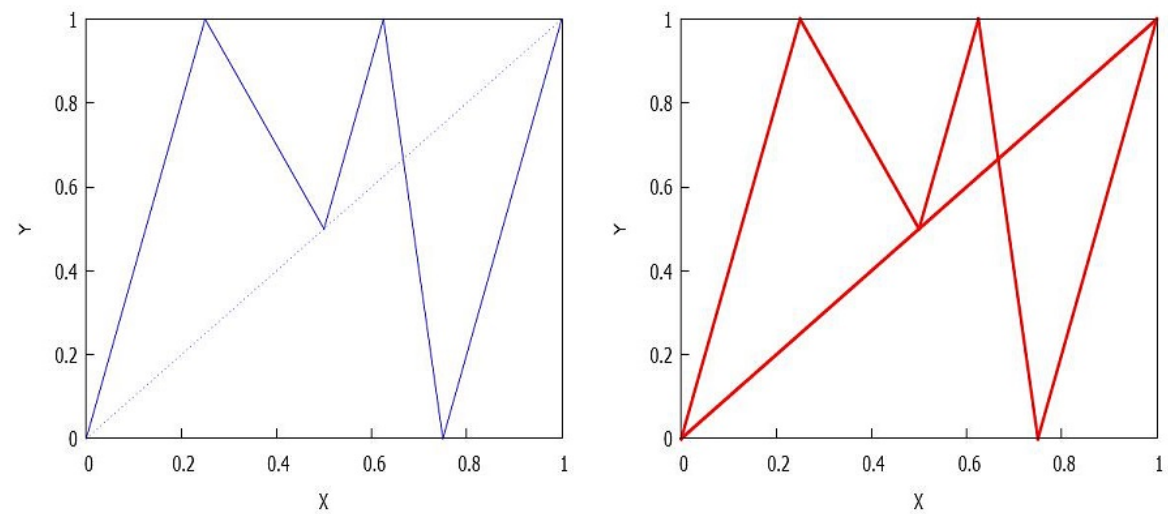

Figure 32. The map $\varphi_{3}$ and the $\omega$-limit set $\omega_{\Phi_{3}}\left(\left(w_{0}, w_{0}\right)\right)$ homeomorphic to $C_{3}$.

In general, for $n=2 k+1, n \geq 5$, we define (see Figure 33 for $n=7$ )

$$
\varphi_{n}(x)= \begin{cases}T\left(2(k-1)\left(x-\frac{j}{2(k-1)}\right)\right), & \text { if } x \in\left[\frac{j}{2(k-1)}, \frac{j+1}{2(k-1)}\right], j=0, \ldots, k-2, \\ \varphi_{3}(2 x-1), & \text { if } x \in\left[\frac{1}{2}, \frac{5}{8}\right] \cup\left[\frac{13}{16}, 1\right], \\ \frac{9}{4}-2 x, & \text { if } x \in\left[\frac{5}{8}, \frac{3}{4}\right], \\ 4 x-\frac{9}{4}, & \text { if } x \in\left[\frac{3}{4}, \frac{13}{16}\right],\end{cases}
$$

where $T(\cdot)$ denotes the well-known tent map $T(z)=1-|1-2 z|, z \in[0,1]$. That is, the graph of $\varphi_{n}(x)$ is composed of $k-2$ tent maps placed between 0 and $\frac{1}{2}$, whereas in $\left[\frac{1}{2}, 1\right]$ we draw the scaled graph of $\varphi_{3}$ in the zones $\left[\frac{1}{2}, \frac{5}{8}\right] \cup\left[\frac{13}{16}, 1\right]$ and try to establish a fixed point in $x=\frac{3}{4}$. Again, if $z_{0} \in I$ is a transitive point of $\varphi_{n}$, and we put $\Phi_{n}(x, y)=\left(y, \varphi_{n}(x)\right)$, it is straightforward to obtain $\omega_{\Phi_{n}}\left(z_{0}, z_{0}\right)=\Delta \cup \Gamma_{\varphi_{n}}$, being $\Gamma_{\varphi_{n}}$ the graph of $\varphi_{n}$.
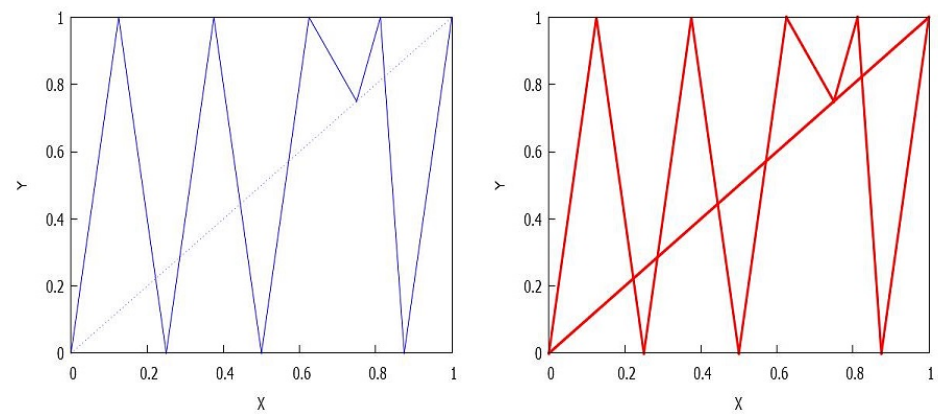

Figure 33. The map $\varphi_{7}$ and the $\omega$-limit set $\omega_{\Phi_{7}}\left(\left(z_{0}, z_{0}\right)\right)$ homeomorphic to $C_{7}$. 
Now, let us present some other connected $\omega$-limit sets whose interiors are empty, and where its aspect is similar to a lattice structure.

Example 1. Consider a totally transitive map $f: I \rightarrow I$, for instance the tent map. Let $x_{0} \in I$ such that $\omega_{f}\left(x_{0}\right)=I$ and define the Cournot map $F(x, y)=(y, f(x))$. On the other hand, take a periodic point $y_{0}$ of period $N \geq 1$. Then, if $\left\{y_{0}, y_{1}, \ldots, y_{N-1}\right\}$ is the periodic orbit, it is easily seen, with the help of Remark 1, that

$$
\omega_{F}\left(x_{0}, y_{0}\right)=\left(I \times\left\{y_{0}, y_{1}, \ldots, y_{N-1}\right\}\right) \cup\left(\left\{y_{0}, y_{1}, \ldots, y_{N-1}\right\} \times I\right) .
$$

In Figure 34 you can see the lattice generated by a periodic point of period 2.

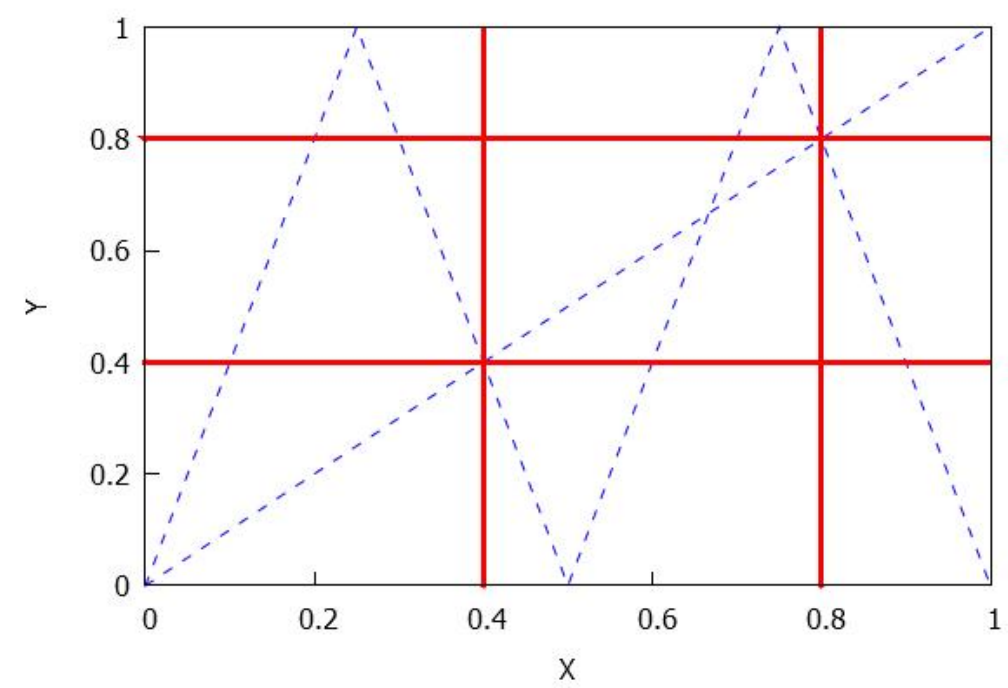

Figure 34. In red, the connected $\omega$-limit set $\omega_{F}\left(x_{0}, y_{0}\right)$ for a periodic point $y_{0}$ of period 2.

According to the aspect of the drawn $\omega$-limit sets of a Cournot map with non-empty interior, we are left the following result concerning the connectivity of such a $\omega$-limit set. Before we state a topological result (see ([31], p. 27)) which implies, as an easy consequence, that the union of two closed sets in the interval has an empty interior if each of them has in turn an empty interior.

Lemma 7. If $K$ is a closed set in a topological space $(X, \mathcal{T})$, and $A \subset X$ is any subset of $X$, then $\operatorname{Int}(K \cup \operatorname{Int}(A))=\operatorname{Int}(K \cup A)$.

We present here a characterization for connected $\omega$-limit sets of Cournot maps that have non-empty interior. Realize that we can justify it in view of our analysis developed in Section 3, nevertheless we give the corresponding theoretical proof.

Proposition 5. Let $F(x, y)=\left(f_{2}(y), f_{1}(x)\right),(x, y) \in I^{2}$ be a Cournot map. Let $\omega_{F}(x, y)$ be an $\omega$-limit set with non-empty interior. Then, $\omega_{F}(x, y)$ is connected if and only if

(1) $\pi_{j}\left(\omega_{F}(x, y)\right)$ is connected, where $\pi_{j}$ is meant the canonical projection to the $j$-th coordinate, $j=1,2$.

(2) $\omega_{F^{2}}(x, y) \cap \omega_{F^{2}}(F(x, y)) \neq \varnothing$.

Proof. Assume that $\omega_{F}(x, y)$ is connected. Since $\pi_{j}$ is continuous, $j=1,2$, we have (1). On the other hand, if $\omega_{F^{2}}(x, y) \cap \omega_{F^{2}}(F(x, y))=\varnothing$, then, taking into account that $\omega_{F}(x, y)=\omega_{F^{2}}(x, y) \cup \omega_{F^{2}}(F(x, y))$, we would have a decomposition of $\omega_{F}(x, y)$ into two closed disjoint sets, which contradicts that $\omega_{F}(x, y)$ is connected.

Now, we assume that (1) and (2) hold, and we are going to prove that in these circumstances $\omega_{F}(x, y)$ is connected. By Proposition 1 and $(1), \pi_{1}\left(\omega_{F}(x, y)\right)=\omega_{f_{2} \circ f_{1}}(x) \cup$ 
$\omega_{f_{2} \circ f_{1}}\left(f_{2}(y)\right)$ is a connected set in the real line, therefore an interval, which is possibly degenerate. If the interval is degenerate, then both $\omega_{f_{2} \circ f_{1}}(x)$ and $\omega_{f_{2} \circ f_{1}}\left(f_{2}(y)\right)$ are singletons (in fact, the same point), and it is easily seen that $\omega_{F}(x, y)$ is reduced to a fixed point, therefore a connected set. So, next we assume that $\pi_{1}\left(\omega_{F}(x, y)\right.$ is a closed interval with nonempty interior. According to Lemma 7, either $\omega_{f_{2} \circ f_{1}}(x)$ or $\omega_{f_{2} \circ f_{1}}\left(f_{2}(y)\right)$ has non-empty interior. Without loss of generality, assume that $\omega_{f_{2} \circ f_{1}}(x)=\bigcup_{i=1}^{m} I_{i}$, where each $I_{i}$ is a nondegenerate subinterval, $I_{r} \cap I_{s}=\varnothing$ if $r \neq s$, and $\left(f_{2} \circ f_{1}\right)\left(I_{i}\right)=I_{i+1(\bmod m)}, i=1, \ldots, m$. Necessarily, either $m=1$ or $m=2$ : if $m \geq 3$, then if we denote $I_{i}=\left[a_{i}, b_{i}\right], i=1, \ldots, m$, the omega-limit set $\omega_{f_{2} \circ f_{1}}\left(f_{2}(y)\right)$ must contain the intervals $\left[b_{i}, a_{i+1}\right]$ for $i=1, \ldots, m-1$, but this is impossible, as was shown in Lemma 4.

(i) If $m=2$, then $\omega_{f_{2} \circ f_{1}}(x)=A=I_{1} \cup I_{2}$, and by force $\omega_{f_{2} \circ f_{1}}\left(f_{2}(y)\right)=I_{1}^{\prime}$, with $\pi_{1}\left(\omega_{F}(x, y)\right)=I_{1} \cup I_{1}^{\prime} \cup I_{2}$. Applying the maps $f_{1}, f_{2}$, we find $\omega_{f_{1} \circ f_{2}}\left(f_{1}(x)\right)=$ $f_{1}\left(\omega_{f_{2} \circ f_{1}}(x)\right)=f_{1}(A)=f_{1}\left(I_{1}\right) \cup f_{1}\left(I_{2}\right)=: J_{1}^{\prime} \cup J_{2}^{\prime}$, and $\omega_{f_{1} \circ f_{2}}(y)=\omega_{f_{1} \circ f_{2}}\left(\left(f_{1} \circ\right.\right.$ $\left.\left.f_{2}\right)(y)\right)=f_{1}\left(\omega_{f_{2} \circ f_{1}}\left(f_{2}(y)\right)\right)=f_{1}\left(I_{1}^{\prime}\right)=: J_{1}$. By Lemma $4, A$ is mixing, so $\left.\left(f_{2} \circ f_{1}\right)^{2}\right|_{I_{i}}$ is topologically mixing, $i=1,2$. Then, by Theorem 2 there exists $\left(x_{1}, y_{1}\right) \in I^{2}$ such that:

(i.1) either $\omega_{F^{4}}\left(x_{1}, y_{1}\right)=\omega_{\left(f_{2} \circ f_{1}\right)^{2}}\left(x_{1}\right) \times \omega_{\left(f_{1} \circ f_{2}\right)^{2}}\left(y_{1}\right)=I_{1} \times J_{1}^{s}$, for some $s \in\{1,2\}$ (in fact, here $J_{1}$ is not mixing for $\left(f_{1} \circ f_{2}\right)$, but we can decompose it as $J_{1}^{1} \cup J_{1}^{2}$ and each $\left.\left(f_{1} \circ f_{2}\right)^{2}\right|_{J_{1}^{j}}$ is mixing, $\left.j=1,2\right)$ : in this case, since $\omega_{F}(x, y)$ has non-empty interior and we can use Lemma 3 , it can be noticed that the we obtain Distribution D6, which contradicts our hypothesis (2) on the intersection of $\omega_{F^{2}}(x, y)$ and $\omega_{F^{2}}(F(x, y))$

(i.2) or $\omega_{F^{4}}\left(x_{1}, y_{1}\right)=\omega_{\left(f_{2} \circ f_{1}\right)^{2}}\left(x_{1}\right) \times \omega_{\left(f_{1} \circ f_{2}\right)^{2}}\left(y_{1}\right)=I_{1} \times J_{1}$, if $\left.\left(f_{1} \circ f_{2}\right)\right|_{J_{1}}$ is mixing; in this case, reasoning as above, we arrive at Distribution D4, see Figure 10, a connected set with non-empty interior.

(ii) Let $m=1$. Notice that if $\operatorname{Int}\left(\omega_{F}(x, y)\right) \neq \varnothing$, also $\omega_{f_{1} \circ f_{2}}(y)$ has non-empty interior, as a consequence of Proposition 1 and the fact that in a Cartesian product the connected components of a set are the product of connected components of each space, see [31]. Here, additionally, we can distinguish two different cases depending on the nature of $\omega_{f_{1} \circ f_{2}}(y)=B$.

(ii.1) If $B=J$ is an interval with $f_{2}(B)=f_{2}(J)=I_{1}$, we obtain: either Distribution D1, with $k=1$, if $I_{1}$ is mixing, therefore a connected $\omega$-limit set holding (1) and (2); or the Distribution that we have found in case $A, B$ no mixing, two rectangles tied by the vertex obtained with the 'middle' points (fixed points) of $I_{1}$ and $J_{1}$.

(ii.2) If $B=J$ is an interval with $f_{1}(A) \cap B \neq \varnothing, f_{1}(A) \cap \operatorname{Int}(B)=\varnothing$, according to Lemma 4 both $A=I_{1}$ and $J_{1}$ are mixing. Reasoning as in Case (i.2), we can find $\left(x_{1}, y_{1}\right)$ such that $\omega_{f_{2} \circ f_{1}}(x)=I_{1}$ and $\omega_{f_{1} \circ f_{2}}(y)=J$, and it is immediate to see that $\omega_{F}$ will be the union of two rectangles tied by a common vertex, a connected set.

Remark 4. We emphasize that the proof strengthens our study in Section 3, because inside it we have found the only possible connected $\omega$-limit sets with non-empty interior that appeared in that section. Moreover, notice that the conditions are always necessary, even in the general frame of a compact metric space and a continuous map $\varphi: X \rightarrow X$ that has the form $\varphi(x, y)=$ $\left(\varphi_{2}(y), \varphi_{1}(x)\right)$, with $\varphi_{j}: X \rightarrow X, j=1$, 2. It remains for us to study whether, in fact, they are or are not also sufficient conditions in order to characterize the connectivity of $\omega$-limit sets of Cournot maps with empty interior.

Remark 5. In the above Proposition, both conditions (1) and (2) are needed in order to attain that the $\omega$-limit set $\omega_{F}(x, y)$ be connected. In this sense, it is possible to find a non-connected $\omega$-limit set $\omega_{F}(x, y)$ holding (1): see Figure 35. 


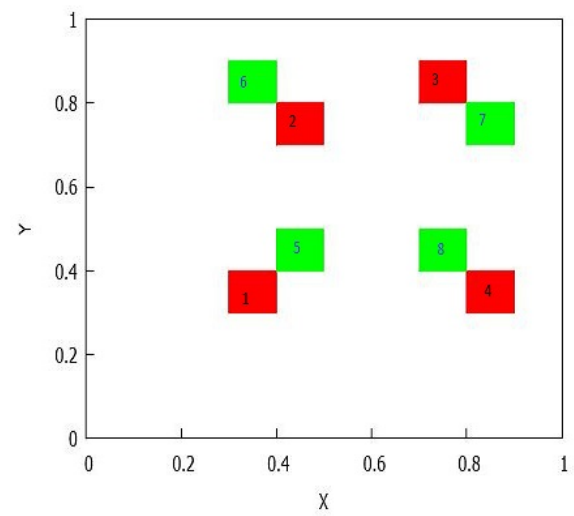

Figure 35. Here, $\omega_{F}(x, y)=W_{1} \cup W_{2}$, with $W_{1}=\omega_{F^{2}}(x, y)$ (in red color) and $W_{2}=\omega_{F^{2}}(F(x, y))$ (in green color); the movement of the orbit by $F$ is $1 \rightarrow 2 \rightarrow 3 \rightarrow 4 \rightarrow 5 \rightarrow 6 \rightarrow 7 \rightarrow 8$; see also Figure 17 .

Additionally, we can find another non-connected $\omega$-limit set $\omega_{F}(x, y)$ verifying, nevertheless, the property (1), see Figure 36 for the non-empty interior case. With regards to the empty interior case, consider the transitive map $f:[0,1] \rightarrow[0,1]$ given by $f(x)=\frac{1}{2}+2 x$ if $0 \leq x \leq 4$, $f(x)=\frac{3}{2}-2 x$ if $\frac{1}{4} \leq x \leq \frac{3}{4}$, and $f(x)=2 x-\frac{3}{2}$ if $\frac{3}{4} \leq x \leq 1$. It is easily seen that $f$ is transitive but not totally transitive. Let $\left\{y_{1}, y_{2}\right\}=\left\{\frac{1}{6}, \frac{5}{6}\right\}$ be a periodic orbit having order two, and define the Cournot map $F(x, y)=(y, f(x))$. If $x_{0}$ has a dense orbit by $f$, so $\omega_{f}\left(x_{0}\right)=[0,1]$, we find $\omega_{F}\left(x_{0}, y_{1}\right)=\left(\left[0, \frac{1}{2}\right] \times\left\{y_{1}\right\}\right) \cup\left(\left\{y_{1}\right\} \times\left[\frac{1}{2}, 1\right]\right) \cup\left(\left[\frac{1}{2}, 1\right] \times\left\{y_{2}\right\}\right) \cup\left(\left\{y_{2}\right\} \times\left[0, \frac{1}{2}\right]\right)$. Then $\omega_{F}\left(x_{0}, y_{1}\right)$ is not connected, however $\pi_{j}\left(\omega_{F}\left(x_{0}, y_{1}\right)\right)=[0,1]$ (see Figure 36 ).
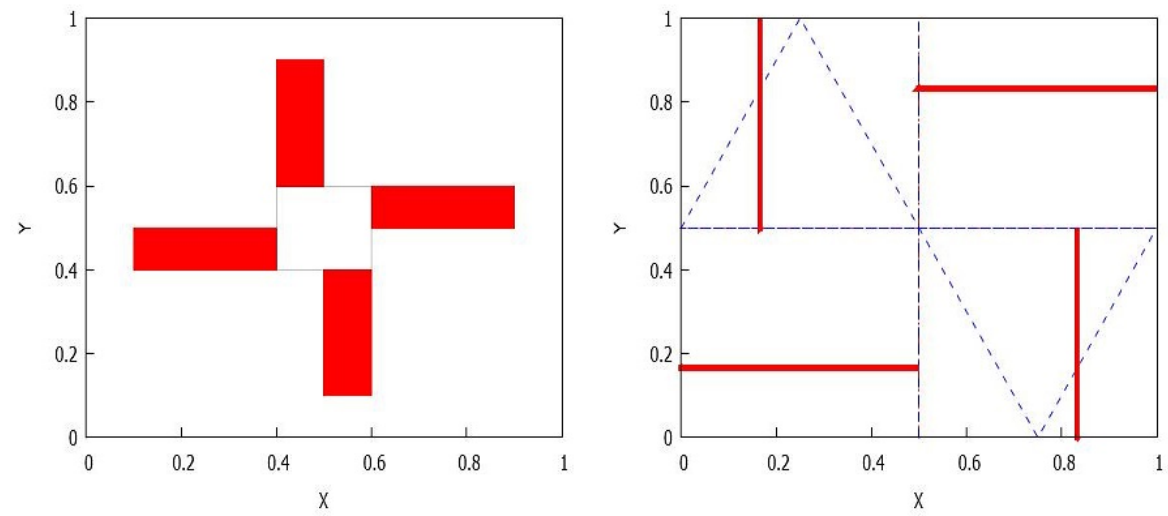

Figure 36. Here, the projections of $\omega_{F}(x, y)$ are connected but $\omega_{F}(x, y)$ is not.

Finally, realize that, according to our description of $\omega$-limit sets of Cournot maps with nonempty interior, we are in a position to assert that both conditions are also sufficient in order to establish the connectivity of $\omega_{F}(x, y)$ in the non-empty interior case, which is an open question in the case of empty interior.

We finish the section by mentioning the notion of minimal set and some associated results, for the case of Cournot maps, extracted from [18]. A minimal set $M \subset I^{2}$ of a Cournot map $F \in C_{A}\left(I^{2}, I^{2}\right)$ is a non-empty, closed and invariant set by $F$, which does not contain a proper subset with the same three properties. In [18] it is proved that a minimal set $M$ of $F$ cannot be of the form $C_{1} \times C_{2}$, where $C_{1}$ and $C_{2}$ are Cantor sets. In the same paper, it is shown that, nevertheless, given two solenoidal sets $Q_{1}$ and $Q_{2}$ having periodic decompositions that are relatively prime, we can find a Cournot map $F(x, y)=(g(y), f(x))$ and $x, y \in I$ such that $\omega_{F}(x, y)=\left(Q_{1} \times f\left(Q_{2}\right)\right) \cup\left(Q_{2} \times f\left(Q_{1}\right)\right)$ is a minimal set of $F$ (for the notion of solenoidal set-a class of Cantor set-the reader is referred to [18] and references therein). An interesting question would be to find, if possible, a Cournot map that has the product $C \times C$ as an admissible $\omega$-limit set, where $C$ is a Cantor set. 


\section{Conclusions}

Despite the difficulty in analyzing $\omega$-limit sets for two-dimensional maps, in the case of Cournot maps we have been able to completely describe the structure of their omega limits with non-empty interior. As a complement, we have shown that these structures arise naturally in some economic models, which can be formulated by Cournot maps, for instance, the Puu's duopoly or the Matsumoto-Nonaka's model. Cyclical phenomena in economics attract great interest. Following Burns and Mitchell (1946), [32], "business cycles are a type of fluctuation found in the aggregate economic activity of nations that organize their work mainly in business enterprises - a cycle consists of expansions occurring at about the same time in many economic activities, followed by similarly general recessions, contractions, and revivals which merge into the expansion phase of the next cycle". In mathematical terms, the orbit of a periodic point is called a cycle of length $n$. In the case at hand, we can observe that replacing points in the orbit by rectangles, a "cyclical movement" applies, in which the rectangle limits the values in each step, thus, a more general cyclical process can be considered and the classification of the orbits with non-empty interior that have been studied throughout this paper contributes to shedding light on economic dynamics.

Obviously, it is an open question to give a complete and detailed description of $\omega$-limit sets of Cournot maps with empty interior. In this paper, we have provided some interesting examples showing that the limit set can be homeomorphic to the circle, even to the union of $k$ circles attached between them by common tangent points; moreover, a difference with the one-dimensional case appears, now the limit set can be connected even though it itself has an empty interior. Another task to be carried out is to find in the non-empty interior case a general formulation for all possible realignments and counting of negative orientations which have appeared in the case $A$ and $B$ no mixing, to try to obtain a closed expression for this number of negative orientations.

Realize that Cournot maps can be generalized in the following way: in the $n$-dimensional cube $I^{n}=[0,1]^{n}$, we define

$$
F\left(x_{1}, x_{2}, \ldots, x_{n}\right)=\left(f_{\sigma(1)}\left(x_{\sigma(1)}\right), f_{\sigma(2)}\left(x_{\sigma(2)}\right), \ldots, f_{\sigma(n)}\left(x_{\sigma(n)}\right)\right),
$$

where $\sigma$ is a (cyclic) permutation of $\{1,2, \ldots, n\}$, and each $f_{\sigma(j)}$ is a continuous function defined from $I$ into itself. These maps can be named permuted direct product maps (see for instance, [33] or [34]). Then, an interesting question would be the study of $\omega$-limit sets with non-empty interior for permuted direct product maps. For instance, for $n=3$, we would have a union of cubes moving in $I^{3}$ according to specific rules to be determined.

Another interesting question is to prove whether the conditions (1) and (2) in Proposition 5 are sufficient or not in order to establish that the corresponding omegalimit is connected. Our conjecture is the affirmative and, even more, we can address the question to the general frame of a product $X_{1} \times X_{2}$ of compact metric spaces and, if we generalize the definition of a Cournot map as $\varphi: X_{1} \times X_{2} \rightarrow X_{1} \times X_{2}, \varphi(x, y)=\left(\varphi_{2}(y), \varphi_{1}(x)\right)$, with $\varphi_{i}: X_{i} \rightarrow X_{j}, i \neq j$, to ask for a characterization of connected $\omega$-limit sets in terms of their projections and the intersection of the omega-limits $\omega_{\varphi^{2}}(x, y)$ and $\omega_{\varphi^{2}}(\varphi(x, y))$.

Author Contributions: Investigation, A.L.-B. and M.M.-G.; Writing-original draft, A.L-B. and M.M.G. The authors contributed equally to this work. All authors have read and agreed to the published version of the manuscript.

Funding: This research was funded by AEI/FEDER, UE, Ministerio de Ciencias, Innovación y Universidades, Spain, Grant number MTM2017-84079-P.

Institutional Review Board Statement: Not applicable.

Informed Consent Statement: Not applicable.

Conflicts of Interest: The authors declare no conflict of interest. 


\section{References}

1. Block, L.S.; Coppel, W.A. Dynamics in One Dimension; Lecture Note in Mathematics 1513; Springer: Berlin, Germany, 1992.

2. Kolyada, S.F.; Snoha, L. Some aspects of topological transitivity—A survey. Grazer Math. Ber. 1997, 334, 3-35.

3. Agronsky, S.J.; Bruckner, A.M.; Ceder, J.G.; Pearson, T.L. The structure of $\omega$-limit sets for continuous functions. Real Anal. Exch. 1989, 15, 483-510. [CrossRef]

4. Bruckner, A.M.; Smítal, J. The structure of $\omega$-limit sets for continuous maps of the interval. Math. Bohem. 1992, 117, 42-47. [CrossRef]

5. Agronsky, S.; Ceder, J. Each Peano subspace of $E^{k}$ is an $\omega$-limit set. Real Anal. Exch. 1991, 17, 371-378. [CrossRef]

6. Agronsky, S.; Ceder, J. What sets can be $\omega$-limit sets in $E^{n}$ ? Real Anal. Exch. 1991, 17, 97-109. [CrossRef]

7. Ceder, J. Some results and problems about $\omega$-limit sets. Real Anal. Exch. 1990, 16, 39-40. [CrossRef]

8. Jiménez López, V.; Smítal, J. $\omega$-limit sets for triangular mappings. Fund. Math. 2001, 167, 1-15. [CrossRef]

9. Kolyada, S.F.; Snoha, L. On $\omega$-limit sets of triangular maps. Real Anal. Exch. 1992, 18, 115-130. [CrossRef]

10. Balibrea, F.; Cánovas, J.S.; Linero, A. $\omega$-limit sets of antitriangular maps. Topol. Appl. 2004, 137, 13-19. [CrossRef]

11. Puu, T. Nonlinear Economic Dynamics, 4th ed.; Springer: Berlin, Germany, 1997.

12. Cánovas, J.S.; Linero, A. Topological dynamic classification of duopoly games. Chaos Solitons Fractals 2001, 12, 1259-1266. [CrossRef]

13. Dana, R.A.; Montrucchio, L. Dynamic complexity in duopoly games. J. Econom. Theory 1986, 40, 40-56. [CrossRef]

14. Kopel, M. Simple and Complex Adjustment Dynamics in Cournot Duopoly Models. Chaos Solitons Fractals 1996, 7, $2031-2048$. [CrossRef]

15. Pražák, P.; Kovárník, J. Nonlinear phenomena in Cournot duopoly model. Systems 2018, 6, 30. [CrossRef]

16. Puu, T. Chaos in Duopoly Pricing. Chaos Solitons Fractals 1991, 1, 573-581. [CrossRef]

17. Rand, D. Exotic phenomena in games and duopoly models. J. Math. Econ. 1978, 5, 173-184. [CrossRef]

18. Balibrea, F.; Cánovas, J.S.; Linero, A. Minimal sets of antitriangular maps. Internat J. Bifur. Chaos Appl. Sci. Engrg. 2003, 13, 1733-1741. [CrossRef]

19. Day, R.H. Irregular growth cycles. Am. Econ. Rev. 1982, 72, 406-414.

20. Friedman, J. Oligopoly theory. In Handbook of Mathematical Economics. Volume 2; Arrow, K.J., Intriligator, M.D., Eds.; North Holland Publishing Co.: Amsterdam, The Netherlands, 1982; pp. 491-534.

21. Friedman, J. Duopoly. In Game Theory; Eatwell, J., Milgate, M., Newman, P., Eds.; Palgrave Macmillan: London, UK, 1989; pp. 133-138.

22. Cournot, A.A. Researches into the Mathematical Principle of the Theory of Wealth; Macmillan: New York, NY, USA, 1838.

23. Puu, T. Chaos in business cycles. Chaos Solitons Fractals 1991, 1, 457-473. [CrossRef]

24. Cánovas, J.S.; Muñoz-Guillermo, M. On the complexity of economic dynamics: An approach through topological entropy. Chaos Solitons Fractals 2017, 103, 163-176. [CrossRef]

25. Cánovas, J.S.; Muñoz-Guillermo, M. On the dynamics of Kopel's Cournot duopoly model. Appl. Math. Comput. 2018, 330, $292-306$. [CrossRef]

26. Matsumoto, A.; Nonaka, N. Statistical dynamics in a chaotic Cournot model with complementary goods. J. Econ. Behav. Organ. 2006, 61, 769-783. [CrossRef]

27. Cánovas, J.S.; Muñoz-Guillermo, M. Describing the Dynamics and Complexity of Matsumoto-Nonaka's Duopoly Model. Abstr. Appl. Anal. 2013, 2013, 18. [CrossRef]

28. Puu, T.; Norin, A. Cournot duopoly when the competitors operate under capacity constraints. Chaos Solitons Fractals 2003, 18, 577-592. [CrossRef]

29. Lupini, R.; Lenci, S.; Gardini, L. Bifurcations and multistability in a class of two-dimensional endomorphisms. Nonlinear Anal. 1997, 28, 61-85. [CrossRef]

30. Sharkovsky, A.N.; Kolyada, S.F.; Sivak, A.G.; Fedorenko, V.V. Dynamics of One-Dimensional Maps; Kluwer Academic Publishers: Dordrecht, The Netherlands, 1997.

31. Engelking, R. General Topology; Heldermand: Berlin, Germany, 1989.

32. Burns, A.F.; Mitchell, W.C. Measuring Business Cycles; NBER: Cambridge, MA, USA, 1946.

33. Linero Bas, A.; Soler López, G. A note on the dynamics of cyclically permuted direct product maps. Topol. Appl. 2016, 203, 147-158. [CrossRef]

34. Linero Bas, A.; Soler López, G. A splitting result on transitivity for a class of $n$-dimensional maps. Nonlinear Dyn. 2016, 84, 163-169. [CrossRef] 